\title{
Cytochrome P450 20A1 in zebrafish: Cloning, regulation and potential involvement in hyperactivity disorders.
}

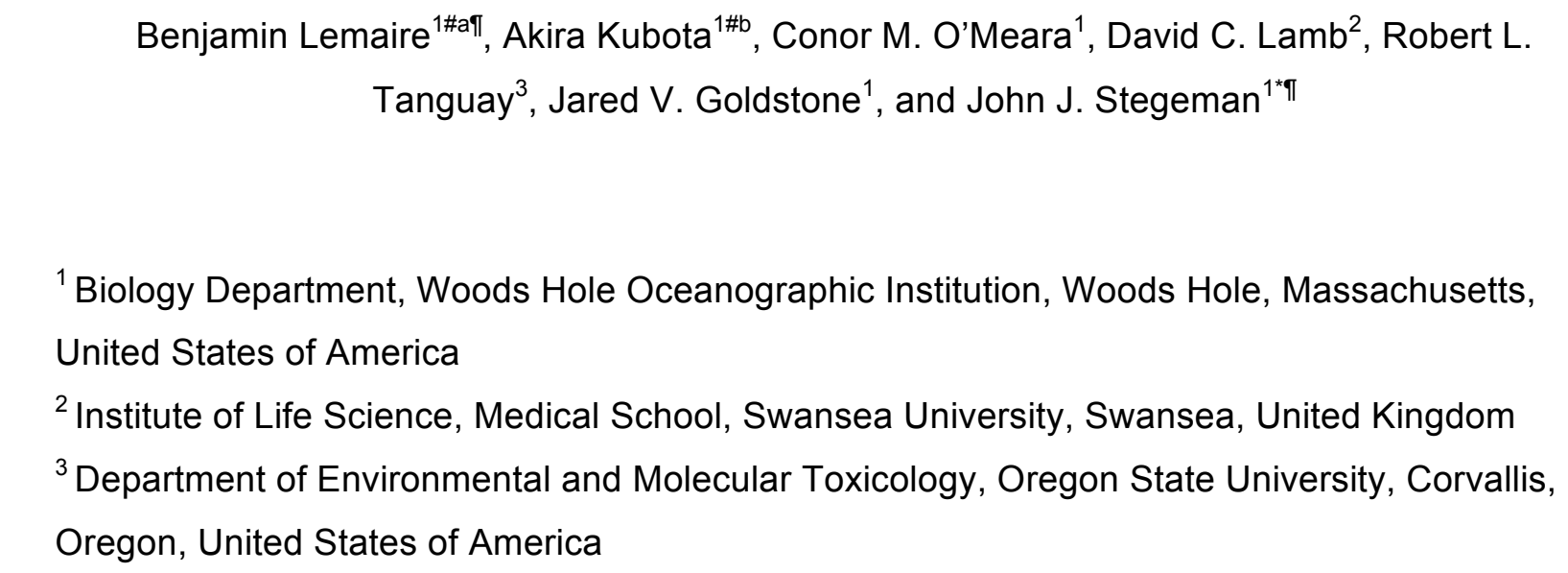

\#a Current address: Institut des Sciences de la Vie, Université Catholique de Louvain, Louvainla-Neuve, Belgium

\#b Current address: Diagnostic Center for Animal Health and Food Safety, Obihiro University of Agriculture and Veterinary Medicine, Obihiro, Japan

I These authors contributed equally to this work.

Number of Tables: 2

4 Number of Figures: 6

Words in Abstract: 247

6

* Corresponding author: John J. Stegeman jstegeman@whoi.edu 


\section{Abbreviations}

34 AHR, aryl hydrocarbon receptor; AR, androgen receptor; arnt2, aryl hydrocarbon receptor

35 nuclear translocator 2; CYP, cytochrome P450; DES, diethylstilbestrol; dpf, days post-

36 fertilization; ef1a, elongation factor 1 alpha; ER, estrogen receptor; ERR, estrogen-related

37 receptor; FXR, farnesoid X receptor; GR, glucocorticoid receptor; HNF4, hepatocyte nuclear

38 factor 4; hpf, hours post-fertilization; LXR, liver X receptor; MeHg, methylmercury; MO,

39 morpholino; OMR, optomotor response; PCB, polychlorinated biphenyl; PPAR, peroxisome-

40 proliferator-activated receptor; PR, progesterone receptor; PXR, pregnane X receptor; qPCR,

41 real-time quantitative PCR; RAR, retinoic acid receptor; ROR, RAR-related orphan receptor;

$42 \mathrm{RXR}$, retinoid X receptor; SF1, vertebrate steroidogenic factor; TF, transcription factor; TL,

43 Tupfel Long-fin zebrafish strain.

44 


\section{Abstract}

45 Cytochrome P450 (CYP) enzymes for which there is no functional information are considered

46 "orphan" CYPs. Previous studies showed that CYP20A1, an orphan, is expressed in human

47 hippocampus and substantia nigra, and in zebrafish (Danio rerio) CYP20A1 maternal transcript

48 occurs in eggs, suggesting involvement in brain and in early development. Moreover,

49 hyperactivity is reported in humans with chromosome 2 microdeletions including CYP20A1. We

50 examined CYP20A1 in zebrafish, including impacts of chemical exposure on expression.

51 Zebrafish CYP20A1 cDNA was cloned, sequenced, and aligned with cloned human CYP20A1

52 and predicted vertebrate orthologs. CYP20A1s share a highly conserved $\mathrm{N}$-terminal region and

53 unusual sequences in the I-helix and the heme-binding CYP signature motifs. CYP20A1 mRNA

54 expression was observed in adult zebrafish organs including liver, heart, gonads, spleen and

55 brain, as well as eye and optic nerve. Putative binding sites in proximal promoter regions of

56 CYP20A1s, and response of zebrafish CYP20A1 to selected nuclear and xenobiotic receptor

57 agonists, point to up-regulation by agents involved in steroid hormone response, cholesterol and

58 lipid metabolism. There also was a dose-dependent reduction of CYP2OA1 expression in

59 embryos exposed to environmentally relevant levels of methylmercury. Morpholino knockdown

60 of CYP2OA1 in developing zebrafish resulted in behavioral effects, including hyperactivity and a

61 slowing of the optomotor response in larvae. The results suggest that altered expression of

62 CYP20A1 might be part of a mechanism linking methylmercury exposure to neurobehavioral

63 deficits. The expanded information on CYP20A1 brings us closer to "deorphanization", that is,

64 identifying CYP20A1 functions and its roles in health and disease.

65

66 Keywords

67 Cytochrome P450 20A1, behavioral disorders, methylmercury, zebrafish 
Introduction

In animals, enzymes of the cytochrome P450 (CYP) superfamily (Nelson, 2009) catalyze

71 oxidation reactions as well as reductions and rearrangements with a vast array of endogenous

72 and exogenous compounds, often with high regio- and stereo-selectivity (Lamb and Waterman,

73 2013). These activities serve a wide range of physiological and toxicological functions. In

74 vertebrate genomes, the number of protein-coding CYP genes range from around 40 to more

75 than 100, in 19 gene families (Nelson et al., 2013). While functions are known for many human

76 CYPs, the physiological substrate(s) and function of a substantial number of vertebrate CYPs

77 remain unknown. In humans, these so-called "orphan P450s" notably include CYP4F22,

78 CYP4V2 and CYP20A1 (Stark and Guengerich, 2007). In the model species zebrafish (Danio

79 rerio), there are few CYPs for which function has been shown. Functions can be inferred for

80 many CYPs in gene families 5-51, based on activities of known mammalian orthologs, yet

81 predicted function has been confirmed in few cases (e.g., the lanosterol $14 \alpha$-demethylase

82 CYP51) (Morrison et al., 2014). In gene families 1-3, there has been much lineage specific

83 expansion, resulting in co-orthologs, most with unknown function (Kubota et al., 2013). Thus,

84 many zebrafish CYPs still are properly considered orphans, including CYP20A1.

85 The sole member of the CYP20 family, CYP20A1, is found in a single copy in human,

86 zebrafish and other vertebrate genomes sequenced to date. The catalytic function of CYP20A1

87 is unknown. Recombinant human CYP20A1 has been tested for activity (Stark et al., 2008),

88 however, no oxidation reaction was detected with several endogenous compounds (steroids,

89 fatty acids, neurotransmitters) or exogenous chemicals (i.e., terfenadine, clotrimazole) that are

90 substrates for some other CYPs. Although limited information is available on this orphan P450,

91 it was found to be relatively highly expressed in human hippocampus and substantia nigra

92 (Stark et al., 2008). These two brain regions are associated with learning and memory, and are

93 involved in neurodegenerative diseases (Wirdefeldt et al., 2011; Zhou et al., 2008). High levels 
94 of CYP20A1 transcript occur also in unfertilized eggs (Goldstone et al., 2010) and in notochord

95 (Thisse and Thisse, 2004) of developing zebrafish, and during embryonic development of

96 mouse (Choudhary et al., 2003).

97 An important observation derives from conditions associated with a microdeletion on

98 chromosome 2 in humans. Patients diagnosed with microdeletions in the $2 q 33$ chromosome

99 region where CYP20A1 is located variously show psychomotor retardation, hyperactivity and

100 bouts of anxiety, among other conditions (Balasubramanian et al., 2011; Tomaszewska et al.,

101 2013). The observations together suggest participation of CYP20A1 early in vertebrate

102 development, and possible involvement in brain functions and behavior. However, these

103 possibilities have not been examined experimentally.

104 In the present study, we employed zebrafish to examine features of CYP20A1 that could

105 bear on its functions. Zebrafish CYP20A1 was cloned and sequenced, and the inferred primary

106 structure was compared to that of the cloned human enzyme, and to CYP20A1 coding

107 sequences found in vertebrate genomes. We determined the organ distribution of transcript in

108 adult zebrafish, and analyzed the transcriptional responses to agonists for several nuclear

109 receptors and the aryl hydrocarbon receptor (AHR) that are prominent in regulating expression

110 of a number of CYP genes in vertebrates (Honkakoski and Negishi, 2000). The latter analysis

111 was complemented by a search for putative binding sites recurring in proximal promoters of

112 vertebrate CYP20A1 genes. We also conducted studies of CYP20A1 mRNA expression in

113 zebrafish embryos with the potent environmental toxicant methylmercury (MeHg). Finally, early-

114 developing zebrafish were screened for morphological and behavioral effects resulting from

115 knockdown of CYP20A1 expression using morpholine-substituted oligonucleotides. The results

116 together provide new information and perspective on the regulation and function of CYP20A1,

117 including possible involvement in neurobehavioral disorders, and the effects of chemicals linked

118 to such disorders.

119 
121 Animals

122 Experiments were conducted on early-developing and sexually mature zebrafish (Danio

123 rerio) of the Tupfel Long-fin ( $T L$ ), Tropical 5D and AB strains, and were approved by the Animal

124 Care and Use Committee of the Woods Hole Oceanographic Institution, Oregon State

125 University and Universite Catholique de Louvain, respectively. The associated Animal Welfare

126 Assurance Numbers are respectively A3630-01, A3229-01 and 1458701. Fish were euthanized

127 by immersion in a bath of tricaine methanesulfonate. Fish maintenance and breeding

128 procedures were previously described (Jonsson et al., 2007a; Jonsson et al., 2007b).

129 cDNA Cloning and Sequencing

130 Primers were designed in the proximal 5' and 3' untranslated regions of zebrafish

131 CYP20A1 transcript [GenBank: NM_213332.1]. Primer sequences were 5'-

132 CTGATGGTCATTGTAGACG-3' (F) and 5'-TCATGGATGTTGGAGTGG-3' (R). We used the

133 Advantage 2 PCR kit from Clontech (Mountain View, USA) to amplify the coding sequence from

$134 \mathrm{TL}$ zebrafish liver cDNA with $1 \mu \mathrm{M}$ of each primer; the thermal profile was: $94^{\circ} \mathrm{C}$ for $1 \mathrm{~min},\left[94^{\circ} \mathrm{C}\right.$

135 for $30 \mathrm{sec}, 58^{\circ} \mathrm{C}$ for $3 \mathrm{~min}$ ] for 35 cycles, and $68^{\circ} \mathrm{C}$ for $7 \mathrm{~min}$. PCR products were purified with

136 the QIAquick Gel Extraction Kit from Qiagen (Valencia, USA) and cloned into pGEM-T Easy

137 from Promega (Madison, USA) as per kit instructions. Mach-1 competent cells from Invitrogen

138 (Carlsbad, USA) were transformed, and, following overnight incubation of selected ampicillin-

139 resistant white colonies in liquid Luria-Bertani medium with antibiotic, plasmids were isolated

140 with the QIAprep Spin Miniprep Kit from Qiagen (Valencia, USA). Three clones were sent to

141 Eurofins MWG Operon (Louisville, USA) for forward and reverse sequencing with T7 primers (1

$142 \mu \mathrm{g}$ of plasmid DNA), and the full-length sequence was then assembled and aligned with

143 [GenBank: NM_213332.1] using MacVector 12.0.2 (Oxford Molecular Group, Madison, USA). 


\section{Secondary structure prediction}

145 Amino acid sequences of CYP20A1 proteins were retrieved from the Ensembl database

146 (Flicek et al., 2013) and aligned with MacVector 12.0.2 software (Oxford Molecular Group, USA)

147 (see Supplemental figure S1 for sequence identifiers). Note that the protein sequence inferred

148 from the TL zebrafish transcript we cloned was fully identical to that predicted from the genome

149 [Ensembl: ENSDARP00000003222]. The specific motifs of CYP20A1 secondary structure (i.e.

$150 \alpha$-helices, $\beta$-strands, transmembrane domain, signal peptide) were predicted with two different

151 online softwares, and overlapping predictions were chosen to construct the consensual

152 secondary structure of the orphan P450. The $\alpha$-helices were predicted with NetSurfP 1.1

153 (Petersen et al., 2009) and Psipred 3.0 (Bryson et al., 2005) at a cut-off of $80 \%$. Putative $\beta$

154 strands were localized with PredictProtein (Rost et al., 2004) and BETApro (Cheng and Baldi,

155 2005) under default settings. Hypothetical transmembrane domains were identified with

156 PredictProtein (Rost et al., 2004) and TMHMM 2.0 (Krogh et al., 2001) at a cut-off of $80 \%$. The

157 possible presence of a signal peptide in the N-terminal portion of CYP20A1 was investigated

158 with pSORTII (Nakai and Horton, 1999) and SignalP 4.1 (Petersen et al., 2011) under default

159 settings. The locations of the P450 signature motifs (i.e., I helix, $\mathrm{K}$ helix and heme-binding

160 region) were analyzed based on the information available in reviews (Sezutsu et al., 2013;

161 Werck-Reichhart and Feyereisen, 2000). The motifs specific to zebrafish and human CYP20A1

162 protein also were compared to those of all other zebrafish and human protein-coding CYPs

163 available in the Ensembl database (data not shown). A subset of the zebrafish CYPs was used

164 as basis for the comparison of CYP20A1 signature motifs shown in Table 1. The sequence

165 identifiers for the selected non-CYP20 isoforms are shown in Supplemental table S6.

166 CYP20A1 heterologous protein expression

167 Zebrafish CYP20A1 was heterologously expressed in E. coli, as before (Barnes et al.,

168 1991). In essence, expression was obtained by replacing the putative membrane anchor N- 
169 terminal region with a membrane anchor from Bos taurus CYP17A1. Preparation of the

170 samples and spectrophotometric analysis of the reduced, CO-ligated protein was done as

171 before (Morrison et al., 2014).

172 In silico promoter analysis

173 The proximal promoter regions of CYP20A1s from the zebrafish, frog, chicken, rat and

174 human were screened to identify putative binding sites for transcription factors (TFs) possibly

175 involved in regulation of the orphan P450. Information on gene sequence was retrieved from the

176 Ensembl database (Flicek et al., 2013). However, in the case of $X$. tropicalis, we used assembly

1777.1 from the Joint Genome Institute available through Xenbase (James-Zorn et al., 2013). A

178 region of $10 \mathrm{~kb}$ upstream of the start codon was selected for analysis with Matlnspector

179 software (Cartharius et al., 2005). The 10kb proximal promoter regions of vertebrate CYP20A1

180 genes were searched with Matlnspector for putative binding sites having either $100 \%$ core

181 sequence identity to known human TF binding sites, or a less stringent $80 \%$ core sequence

182 identity. The putative TF binding sites that occurred in common in the different species'

183 CYP20A1s were classified in two groups, one for nuclear receptor TFs and the other for non-

184 nuclear receptor TFs. Data for the latter group were subjected to an additional analysis. That is,

185 the Uniprot database (Bairoch et al., 2005) was interrogated to identify recurring gene ontology

186 terms in the pool of these recurring non-nuclear receptor TFs. Gene ontology information was

187 retrieved for rat and human TFs whenever possible. The database generated was interrogated

188 to identify the cell types, biological systems and processes over-represented in this pool of non-

189 nuclear receptor TFs.

190 Organ distribution

191 Transcript abundance was analyzed in various organs of TL zebrafish by means of

192 quantitative real-time PCR (qPCR), using liver as a reference tissue. The protocol of fish

193 dissection followed that of Gupta \& Mullins (Gupta and Mullins, 2010), using MS-222 for 
194 euthanasia. For the first set of samples, 12 males and 12 females were dissected to obtain liver,

195 intestine, gonad, heart, kidney, whole brain and eye; organs were pooled from four males and

196 from four females, giving three pools for each sex. Statistical analyses were conducted on

197 tissues of males and females separately. For the second set, 12 males were dissected and

198 tissues pooled to obtain three pools each of liver, spleen, forebrain, midbrain, hindbrain and

199 optic nerve. All samples were flash-frozen in liquid nitrogen upon collection and stored at $-70^{\circ} \mathrm{C}$

200 until processing. RNA was extracted with the Aurum Total Fatty and Fibrous Tissue kit from Bio-

201 Rad (Hercules, USA) as per kit instructions. Total RNA was quantified by spectrophotometry

202 and purity checked with a Nanodrop ND-1000 (Wilmington, USA) at $\mathrm{OD}_{260 / 280}$ and $\mathrm{OD}_{260 / 230}$.

203 Reverse transcription of $1 \mu \mathrm{g}$ (first set) or $150 \mathrm{ng}$ (second set) of total RNA was done with the

204 iScript cDNA Synthesis kit from Bio-Rad, as per kit instructions. QPCR were run on a MylQ

205 single-color real-time thermocycler with IQ Sybr green Supermix from Bio-Rad. The $E^{-\Delta \Delta C t}$

206 method was used for relative quantification (Livak and Schmittgen, 2001), using the geometric

207 mean of aryl hydrocarbon receptor nuclear translocator 2 (arnt2) and elongation factor 1 alpha

208 (ef1 $\alpha)$ Ct values for normalization (Kubota et al., 2013; Morrison et al., 2014). Primers were: 5'-

209 TACAGGAGGTGGAAGGAAAGGTG-3' (F) and 5'-GACGACCACCAAGGGCATAGATAAC-3'

210 (R) for CYP20A1.

211 Exposure to nuclear and cytosolic receptor agonists, and methylmercury

212 Experimental exposures of zebrafish to selected nuclear and xenobiotic receptor

213 agonists were conducted on pools of $15 \mathrm{TL}$ embryos, in $60 \mathrm{ml}$ of $0.3 X$ Danieau's solution.

214 Exposures began at 24 hours post fertilization (hpf), and lasted $24 \mathrm{~h}$ (up to $48 \mathrm{hpf}$ ) or $48 \mathrm{~h}$ (up to

$21572 \mathrm{hpf}$ ). Tested chemicals in solvent carrier (dimethylsulfoxide or ethanol) or solvent carrier

216 alone were added at a final volume of $0.1 \%$. Supplemental table S2 provides a list of the tested

217 chemicals and their known action on nuclear or xenobiotic receptors in mammals and zebrafish,

218 to the best of our knowledge. Ethanol was used as solvent only for exposures to a-tocopherol,

219 arachidonic, docosahexaenoic and eicosapentaenoic acids. Exposures to photosensitive 
220 agonists were conducted for $24 \mathrm{~h}$ at nominal concentrations of $0.1-1-10 \mu \mathrm{M}$. Other

221 exposures were conducted for $48 \mathrm{~h}$ at $0.1-1-3.2 \mu \mathrm{M}$, except that PCB 126 was tested for 48

222 h at doses $(1-10-32 \mathrm{nM})$ that elicit CYP1 induction in zebrafish without compromising viability

223 (Jonsson et al., 2007a; Jonsson et al., 2007b). All chemicals were of $\geq 95 \%$ purity. Following

224 incubations, TL embryos were quickly washed with 0.3X Danieau's solution, frozen in liquid

225 nitrogen and stored at $-70 C^{\circ}$ until RNA extraction. Sample preparation and qPCR analysis were

226 the same as used in the tissue distribution analysis, except that extraction was performed with

227 Aurum Total RNA Mini kit. One $\mu \mathrm{g}$ of total RNA was used for reverse transcription.

228 Experimental exposures of zebrafish to methylmercury (MeHg) chloride were conducted

229 on pools of $15 \mathrm{AB}$ embryos, in $60 \mathrm{ml}$ of $1 \mathrm{X} \mathrm{E3}$ medium, at the Institute of Life Sciences

230 (Universite Catholique de Louvain). Exposure to 10 and $50 \mu \mathrm{g} \mathrm{I}^{-1} \mathrm{MeHg}$ began at $24 \mathrm{hpf}$ and

231 embryos were sampled at 48 hpf, a protocol that does not result in embryo mortality (Hassan et

232 al. 2012). Solvent carrier was distilled water (0.1\% final volume). Following incubations, AB

233 embryos were washed with E3 medium, frozen in liquid nitrogen and stored at $-70^{\circ} \mathrm{C}$ until RNA

234 extraction. We used the exact same procedure of RNA extraction and cDNA synthesis as for TL

235 embryos above. The qPCR experiments were run on a StepOne Plus real-time thermocycler

236 with SYBR Select Master Mix, both from Applied Biosystems. We confirmed that this change of

237 qPCR protocol did not affect the specificity or efficiency of amplification using the same

238 reference genes and method of relative quantification as in TL exposure studies above.

239 Morpholino-based CYP20A1 knockdown

240 Fertilized zebrafish eggs were injected at the 2- to 4-cell stage with fluorescein-tagged

241 morpholino oligonucleotides (MO) from Gene Tools (Philomath, USA), complementary to a

242 region encompassing the CYP20A1 start codon (5'-AATCTAGCATTTTTGCTTTTCAGGGA-3').

243 Morpholino knockdown is transient but effects can persist to 5 or more days post fertilization.

244 Controls for the specificity of gene knockdown consisted of embryos injected with a control MO 
245 (5'-CСTCTTACCTCAGTTACAATTTATA-3'), and non-injected embryos. Hence, three groups

246 were defined for phenotyping studies, non-injected, control MO-injected, and CYP20A1 MO-

247 injected fish. Microinjection of $2.1 \mathrm{nl}$ of $0.18 \mathrm{mM} \mathrm{MO}$ followed the protocol previously described

248 (Jonsson et al., 2007a). The efficacy of CYP20A1 MO to block translation of the CYP20A1 was

verified by inhibition of in vitro protein synthesis with a TnT assay from Promega (the inhibition

of in vitro synthesis was about 50\%; see Supplemental Figure S2). We used only those

embryos showing high-level fluorescence at $24 \mathrm{hpf}$, indicating abundant $\mathrm{MO}$ in the embryo.

For the Optomotor and Movement Tracking behavioral studies with the TL strain

(described below), larvae were taken from each separate clutch or batch of TL zebrafish and

distributed into three groups (i.e., in non-injected, control MO-injected, and CYP20A1 MO-

injected groups), with 30 individuals in each group. Initially, early-developing zebrafish were

kept in 0.3X Danieau's solution for three days and $72 \mathrm{hpf}$ embryos were then transferred in

system water. Larvae from each group were fed once at 4 days post-fertilization (dpf) with a

mixture of spirulina and 30- $\mu$ m Hathchfry Encapsulon diet from Argent (Redmond, USA).

Behavior assays were conducted at $5 \mathrm{dpf}$. TL fish in the various groups were examined daily by

microscopy for any morphological abnormalities

Optomotor assay

Six separate clutches or batches of TL zebrafish larvae were used to study the optomotor response (OMR). With each batch, OMR was assessed on 20 larvae from each of the non-injected, control MO-injected, and CYP20A1 MO-injected groups. The 20 larvae were randomly selected from the 30 larvae in each group. A transparent test chamber $(25 \mathrm{~cm}$ long), placed on top of a computer monitor, was filled with system water at $26.5 \pm 1.5^{\circ} \mathrm{C}$. A pattern of

267 black and white stripes, moving with a period of $6.5 \mathrm{~cm}$ and a frequency of $0.5 \mathrm{~Hz}$, was

268 displayed on the monitor. Larvae were assayed individually. Since zebrafish respond to low

269 spatial frequencies by low velocities of optomotor response (Maaswinkel and Li, 2003),

270 individuals were tested over 1-min periods and distances travelled were recorded every $15 \mathrm{~s}$. 
271 There were no signs of aliasing under these conditions, and the responsiveness and distance

272 travelled by control larvae were not significantly increased when the frequency of wave motion

273 was doubled (data not shown). All trials were conducted between 3:00 and 7:00 PM, and larvae

274 from one group were tested before those from the other two groups. To rule out a possible

275 effect of time on the optomotor responses, three batches were tested, with the non-injected

276 controls tested first and the CYP20A1 MO fish tested last, and three others were conducted in

277 the reverse order. Similar patterns of OMR were observed in all experiments.

278 Movement tracking and activity quantitation

279 Behavioral studies were also conducted with TL zebrafish, using an automated video

280 tracking system from ViewPoint Life Sciences (Montreal, Canada). For each experimental group

281 (i.e., non-injected, control MO-injected, and CYP20A1 MO-injected), 8 larvae were randomly

282 selected from among the 10 individuals that were not tested in the OMR assay. These larvae

283 were transferred individually in the wells of a 24-well plate filled with $2 \mathrm{ml}$ of system water (26.5

$\left.284 \pm 1.5^{\circ} \mathrm{C}\right)$. Motion and activity were recorded in consecutive 10-min videos under white light

285 illumination. The first session (tracking) was started 5 min after larvae were introduced in the

286 plate. The second session (activity) was started 5 min after completion of the first recording. For

287 the tracking experiment, thresholds were: $0.5 \mathrm{~cm} . \mathrm{s}^{-1}$ for large movement and $0.1 \mathrm{~cm} . \mathrm{s}^{-1}$ for

288 inactivity. For activity analysis, thresholds were 10 pixels per frame for burst and 1 for freeze.

289 High-throughput Morphological assessment and Photomotor response control MO-injected and CYP20A1 MO-injected Tropical 5D strain zebrafish at the Department of Environmental and Molecular Toxicology, Oregon State University. For this study, at 4 hours 
individually transferred to the wells of a 96 -well plate with $100 \mu$ l of embryo medium at $28^{\circ} \mathrm{C}$.

Plates were sealed and protected from light for up to $120 \mathrm{hpf}$ (Truong et al., 2014). Tropical 5D

zebrafish from each of the three experimental groups also were assessed at $24 \mathrm{hpf}$ for

photomotor response according to previously published procedures (Truong et al., 2014).

300 Statistical analyses

301 Statistical analyses for results obtained with the TL and AB strains were conducted at

302 the $\alpha=0.05$ level of significance with the Statistica 7.1 package from Statsoft (Tulsa, USA).

303 Data were tested for normality by Kolmogorov-Smirnov test, and for homoscedasticity by

304 Levene's test. Since multiple independent experiments were necessary to investigate CYP20A1

305 expression at all doses of each chemical in exposure studies (except for MeHg), qPCR data

306 were compared by two-tailed Student's t-test with batch-specific solvent controls. The MeHg

307 dose-response data were analyzed by one-way ANOVA followed by post hoc LSD Fisher's test.

308 The non-parametric Kruskal-Wallis ANOVA, followed by Z-adjusted Mann-Whitney U test, was

309 used for statistical analyses of the tissue distribution and OMR studies. Data for movement

310 tracking and activity quantitation of TL larvae were analysed by one-way ANOVA followed by

311 post-hoc LSD Fisher's test. Results were expressed either as mean \pm SEM or mean \pm SD, and

312 graphs generated with Sigma Plot 8.0 software from Systat (San Jose, USA). Statistical

313 analyses for results with Tropical 5D fish were conducted as before (Truong et al., 2014).

\section{Results}

316 Cloning and sequence analysis of zebrafish CYP20A1

317 Primers designed to the proximal 5' and 3' untranslated regions of zebrafish CYP20A1

318 transcript were used to amplify a full-length coding sequence from the Tupfel Long-fin (TL)

319 strain. A protocol with a low annealing temperature was selected to ensure that possible strain-

320 specific polymorphism in the untranslated regions did not impede primer binding and PCR 
321 efficiency. A bright band of the expected size was obtained, and results of sequencing revealed

322 that the transcript from the TL strain was fully identical to the cDNA sequence of zebrafish

323 CYP20A1 available in various databases (GenBank: NM_213332.1; Ensembl:

324 ENSDART00000019325). The inferred amino acid sequence of our cloned zebrafish CYP20A1

325 shares sequence identities ranging from 59-66 \% with CYP20A1 found in genomes of tetrapods,

326 and from $69-82 \%$ with CYP20A1 from genomes of other fishes (Supplemental table S1). Such

327 percentages were to be expected for members of a given vertebrate P450 subfamily.

328 Sequence structure and P450 signature motifs

329 The amino acid sequence derived from the cloned zebrafish CYP20A1 and the cloned

330 human CYP20A1 (Stark et al., 2008) are aligned in Fig. 1. Features of protein structure that are

331 alpha-helices $(n=13)$, beta strands $(n=8)$ and P450 signature motifs $(n=3$; see Table 1

332 headings) were identified based on alignment with other protein-coding CYPs from human and

333 in silico predictions. As shown in Fig. 1, a conserved N-terminal segment of about 30 residues

334 that comprises a transmembrane domain typical of microsomal P450s is found in human and

335 zebrafish CYP20A1s. A signal peptide -perhaps targeting the protein to mitochondria- also is

336 predicted to occur, just after the transmembrane domain. P450 signature motifs are identified in

337 the I-helix, the $\mathrm{K}$ helix and the heme-binding region. However, the amino acid sequences in two

338 of these motifs have features that are unusual compared to most other animal CYPs. Thus, the I

339 helix signature of zebrafish CYP20A1 (aa 276-281, AGCVIT) diverges at the fourth and fifth

340 positions, notably lacking the usually conserved Thr at the fifth position, which is involved in the

341 protonation of oxygen during the P450 catalytic cycle (Sezutsu et al., 2013). The heme-binding

342 region of the zebrafish CYP20A1 (aa 403-411, FSGSQACPE) is one residue shorter than in

343 most P450s, and displays an unusual sequence around the conserved Cys that serves as the

344 apical heme iron ligand. Similar divergence from typical P450 motifs is seen in the I-helix and

345 heme binding peptide of human CYP20A1.

346 The amino acid sequences of the cloned zebrafish and human CYP20A1s also were 
347 aligned with the CYP20A1s of selected other vertebrate species, gleaned from genome

348 databases (Supplemental figure S1). This alignment showed the presence of a conserved N-

349 terminal segment and the unusual sequence features of the I-helix and the heme binding motifs

350 in these other species, similar to those in zebrafish and human.

351 We compared the amino acid sequences of the three signature motifs in zebrafish

352 CYP20A1 to those in other zebrafish CYP families (Table 1). Divergence at the fourth and fifth

353 positions of the I helix motif like that in CYP20A1 appears as well in CYP7A1 (cholesterol $7 \alpha-$

354 hydroxylase), CYP8A1 (prostacyclin synthase), CYP8B1 (sterol 12 $\alpha$-hydroxylase), and several

355 related zebrafish orphan CYPs (i.e., CYP7C1, CYP8B2 and CYP8B3), as well as CYP39A1

356 (oxysterol $7 \alpha$-hydroxylase). Thus, the fourth position of the I-helix motif in all of these P450s has

357 a hydrophobic rather than an acidic residue, and the fifth position does not contain a Thr

358 residue. Instead, an Asp residue is found in all of the above-mentionned CYPs except CYP20A1

359 (which has an lle residue). Most of these other CYPs do have a Thr at the $6^{\text {th }}$ position, raising a

360 question about whether that Thr might fulfill a role in proton transfer to the oxygen. However,

361 CYP8A1 and CYP39A1 do not have a Thr at either the $5^{\text {th }}$ or the $6^{\text {th }}$ positions.

362 Divergence from the classical motif of the heme-binding region was also identified for

363 CYP7A1, CYP8A1, CYP8B1, CYP39A1 (Table 1), and the related CYP7 and CYP8 orphans.

364 However, these sequences were not identical to that seen in CYP20A1, which has a unique

365 heme-binding region. Thus, among zebrafish P450s, CYP20A1 is the only isoform with one

366 residue missing at the beginning of the motif, a glutamine (neutral residue) instead of a basic

367 residue at the center of the motif, and a glutamic acid instead of a glycine (non polar residue) at

368 the terminal position. The same features distinguish the CYP20A1 from other P450s in human.

369 CYP20A1 heterologous protein expression

370 Zebrafish CYP20A1 was heterologously expressed in E. coli to determine whether a

371 protein with a native P450 spectrum could be obtained, given the unusual sequence features. A 
372 reduced CO-difference spectrum typical of P450 was obtained (Fig. 2), which indicates

373 expression of native protein. The presence of a peak at $420 \mathrm{~nm}$ in the CO-difference spectrum

374 indicates partial loss of the cysteine thiolate linkage to the heme, which is sometimes the result

375 of protein misfolding of non-native (heterologous) proteins in E. coli. However, we also observed

376 that the $450 \mathrm{~nm}$ peak was rapidly converted to $420 \mathrm{~nm}$, which suggests that the ferrous - CO

377 complex is unstable, a condition observed with prostacyclin synthase (CYP8A1), an enzyme

378 that acts on substrates that carry their own oxygen (Yeh et al., 2005). We did examine the

379 expressed protein for Type-1 (substrate) binding speotra with cholesterol and arachidonic acid,

380 but these preliminary studies failed to detect any binding to recombinant zebrafish CYP20A1

381 (authors' unpublished information).

382

Tissue distribution and developmental expression of CYP20A1

Real-time quantitative PCR (qPCR) analysis was used to determine levels of CYP20A1

384 transcript in organs from male and female zebrafish. Two sets of samples were analyzed, with

385 tissues pooled from multiple individuals. In the first set, both males and females were examined.

386 In females, there was a significantly higher level of expression in ovary and heart than liver,

387 intestine, kidney, brain and eye (Fig. 3A). In corresponding male samples, transcript levels in

388 testis were higher than in the other tissues examined, and levels of expression were lower in

389 heart and eye than in liver or brain (Fig. 3B). In the second set, we examined organs from male

390 only. Expression levels were compared in different parts of the brain (forebrain, midbrain and

391 hindbrain), optic nerve, spleen and liver. CYP20A1 transcript was slightly more abundant in

392 spleen and optic nerve than in the other tissues (Fig. 3C).

393 Earlier we had reported on the levels of most CYP transcripts during zebrafish

394 development, covering the period from 3 to 48 hours hpf (Goldstone et al., 2010). However, the

395 levels of CYP20A1 were not readily discernible in the figures in that report. Values for CYP20A1

396 were extracted from the previous microarray data, showing that CYP20A1 transcript was more

397 abundant in embryos during the segmentation period (12-24 hpf) (Supplemental Figure 3). 
Reponses to chemical treatment

Possible effects on CYP20A1 expression were assessed in zebrafish embryos that had been exposed to agonists for selected nuclear and xenobiotic receptors. Emphasis was put on agonists for receptors involved in steroid function (androgen, estrogen and glucocorticoid

402 receptors - AR, ER, and GR respectively), cholesterol and lipid metabolism (liver X, farnesoid X 403 and peroxisome-proliferator-activated receptors - LXR, FXR, and PPAR respectively), response

404 to xenobiotics (pregnane $\mathrm{X}$ receptor, PXR, and the aryl hydrocarbon receptor, AHR), and 405 morphogenesis (retinoic acid receptor RAR) (see Supplemental table S2). Dose-response

406 studies with these chemicals were conducted in zebrafish embryos with exposure beginning at

\begin{tabular}{l|l}
407 & $24 \mathrm{hpf}$, and sampling at 48 or $72 \mathrm{hpf}$ (i.e., exposure for 24 or $48 \mathrm{~h}$ ). Significant but modest
\end{tabular} 408 increases of transcript abundance (about $150 \%$ of solvent controls) were observed in embryos 409 exposed for $48 \mathrm{hr}$ to $1 \mu \mathrm{M}$ of diethylstilbestrol (Fig. 4A), a known ER agonist in zebrafish, or 410 T0901317 (Fig. 4B), a known LXR-FXR agonist in zebrafish. At the highest dose, farnesol 411 isomers (FXR agonists in zebrafish) and pregnenolone (PXR agonist in zebrafish) also led to a 412 mild increase (i.e., about $30 \%$ ) of CYP20A1 mRNA content in embryos (48 h exposure) (Fig. 413 4B). A similar increase was detected in embryos exposed to $1 \mu \mathrm{M}$ dexamethasone (Fig. 4A), a

414 GR agonist in zebrafish. Slight yet significant increases of expression (i.e., 10-20\%) were also 415 observed with $10 \mu \mathrm{M}$ of docosahexaenoic acid (24 h exposure) (Supplemental table S3), a

416 PPAR agonist in zebrafish, $1 \mu \mathrm{M}$ of pregnenolone and $3.2 \mu \mathrm{M}$ of the AR agonist

417 androstenedione (48 h exposures) (Fig. 4). Expression of CYP2OA1 decreased by about 10-

$41820 \%$ in embryos exposed to doses up to $3.2 \mu \mathrm{M}$ all-trans retinoic acid (24 $\mathrm{h}$ exposures)

419 (Supplemental table S3), a RAR agonist in zebrafish. (At $10 \mu \mathrm{M}$, all-trans retinoic acid treatment 420 resulted in $98 \%$ mortality.)

421 We also examined response to the non-ortho-substituted (dioxin-like) polychlorinated 422 biphenyl (PCB) 126, an AHR agonist (Supplemental table S2). Exposures were for $48 \mathrm{~h}$, from 42324 to $72 \mathrm{hpf}$. Nanomolar doses of PCB 126, which are highly effective at inducing the target 
424 gene CYP1A in zebrafish (Jonsson et al., 2007b), did not elicit any change in CYP20A1

425 expression (not shown).

426 To assess further the possible involvement of nuclear receptors and other transcription

427 factors (TFs) in the regulation of CYP20A1 expression, we used an in silico search to predict

428 putative TF binding sites. A $10 \mathrm{~kb}$ proximal promoter region of the zebrafish and human

429 CYP20A1 genes was examined, along with the promoters of CYP20A1 predicted in genomes of

430 several other vertebrate species (rat, chicken, frog). We screened these regions for the

431 presence of possible binding sites (i.e., response elements) for nuclear and xenobiotic

432 receptors. When restricting the search to putative binding sites exactly matching the human

433 core sequences, several were found to occur in proximal promoters of all the vertebrate

434 CYP20A1 genes examined. These response elements are for the vertebrate steroidogenic

435 factor 1 (SF1), the estrogen receptors and estrogen-related receptors (ERs and ERRs), the

436 retinoic acid receptor-related orphan receptor alpha (ROR $\alpha)$, the peroxisome proliferator-

437 activated receptors (PPARs) and glucocorticoid receptor (GR) (Table 2). When a less stringent

438 cut-off of core sequence similarity was applied (i.e., $80 \%$ ), response elements were identified

439 also for other nuclear receptors. These include the androgen receptor (AR), the progesterone

440 receptor $(\mathrm{PR})$, the farnesoid $\mathrm{X}$ receptor (FXR), the hepatocyte nuclear factor 4 (HNF4) and the

441 retinoid receptors (RAR and retinoid X receptor - RXR) (Supplemental table S4). At 70\% core

442 sequence identity, binding sites were identified for the liver X receptor (LXR) but not AHR (data

443 not shown).

444 Proximal promoter analyses also identified putative binding sites for numerous TFs that

445 were not nuclear or xenobiotic receptors. Gene ontology terms were compiled from the Uniprot

446 database (Bairoch et al., 2005) for these recurring TFs, and keyword searches conducted to

447 determine the proportion of TFs putatively involved in CYP20A1 regulation that were associated

448 with a particular cell type, biological system or process. At $100 \%$ core sequence identity, TFs

449 active in the brain, blood cells (among which $77 \%$ were notably involved in immunity; data not 
450 shown), skeletal system, eye, sensory system, germ cells and stem cells were over-

451 represented, as were TFs involved in neurogenesis, cell migration, neurulation and

452 anterior/posterior patterning. Wingless (Wnt), Smoothened and Notch were the major

453 developmental signaling pathways represented (Supplemental Table S5). Similar observations

454 (i.e., enrichment of ontology terms) were made at $80 \%$ core sequence identity (data not shown).

455 To assess the influence of environmental toxicants on CYP20A1 expression, we

456 examined zebrafish embryos exposed to MeHg chloride (10 and $50 \mu \mathrm{gg} . \mathrm{I}^{-1}$ ) from 24 and $48 \mathrm{hpf}$

457 (Hassan et al., 2012). The MeHg elicited dose-dependent reduction in CYP20A1 mRNA levels

458 in zebrafish embryos (Fig. 5). With the higher dose tested, CYP20A1 transcript abundance was

459 less than half the level in solvent controls, and was significantly lower than the levels obtained

460 with a $10 \mu \mathrm{g} \cdot \mathrm{I}^{-1}$ dose $(p=0.048$; not shown $)$.

461 Knockdown of CYP20A1 and phenotypic effects

462 To gain insight into the function of CYP20A1 protein in zebrafish, we used a morpholino

463 (MO) gene knockdown approach. Morpholinos targeted to the CYP20A1 translation start site,

464 which inhibits CYP20A1 protein synthesis, were injected into 2- to 4-cell stage embryos and

465 morphological and behavioral phenotypes screened at various times. In these studies we used

466 two approaches, with different strains, for assessing morphological and behavioral effects of MO

467 treatment. With TL zebrafish, morphology was assessed daily daily, and no obvious effects

468 were seen in fish that were uninjected, control MO-injected or CYP20A1 MO-injected.

469 Morphology was examined also in a high-throughput analysis using the Tropical 5D wild-type

470 strain. In those latter analyses embryos injected with coded CYP20A1 or control MOs, and non-

471 injected controls, were examined in a blind test at $24 \mathrm{hpf}$ and at five days post-fertilization (dpf).

472 At $24 \mathrm{hpf}$, somites and notochord appeared normal in both control MO and CYP20A1 MO

473 embryos. Viability was unaffected, however, CYP20A1 MO-injected embryos exhibited a

474 delayed development (data not shown). There were no morphological abnormalities noticed at 5

$475 \mathrm{dpf}$ in either the control MO or the CYP20A1 MO groups, i.e., yolk sac, body axis, eye, snout, 
476 jaw, otic vesicle, heart, brain, fins, trunk, swim bladder, notochord, pigmentation and circulation

477 appeared normal.

of development, $24 \mathrm{hpf}$. For this assay, we employed the Tropical 5D strain injected with coded

CYP20A1 MO or control MO as above, and movement (tail flexion) was examined in ovo

(Truong et al., 2014). Analysis revealed a greater in ovo activity in the CYP20A1 MO treated embryos, in both the dark-baseline and light-excitatory phases (Figure 6).

For later stage behavioral studies, TL strain zebrafish (the strain from which CYP20A1 had been cloned) were injected with control or CYP20A1 MOs at the 2- to 4-cell stage and at five dpf were screened for an optomotor response, and for locomotory activity with an automated tracking system. Non-injected TL zebrafish embryos were similarly tested.

We first tested for the optomotor response (OMR), a test for visual responsiveness in which fish larvae follow waves of vertical black and white stripes displayed on the fish tank bottom. Larvae from the three experimental groups were tested individually over 1-min periods, 490 and the distance they travelled was recorded every $15 \mathrm{~s}$. On average, larvae in the CYP20A1 491 MO group travelled about $20 \%$ less distance than those injected with control MO, or not injected 492 (Fig. 7A). A substantial number of larvae in the CYP20A1 MO group did not initiate an oriented movement after 15 or $30 \mathrm{~s}$ of stimulation, indicating latency or reduced responsiveness of these

494 fish to the visual stimulus. The CYP20A1 MO treatment did not appear to affect the locomotory 495 capacity of larvae that did respond to the stimulus, based on distances traveled per unit time 496 once the response began.

497 We then investigated the spontaneous locomotory behavior of CYP20A1 MO-injected TL 498 larvae, using an automated tracking system (Viewpoint Zebrabox). In three independent 499 experiments, eight larvae from each group (CYP20A1 MO-injected, control MO-injected and, 500 non-injected) were tested simultaneously in 24-well plates, under constant illumination. Two 10501 min movies were recorded, one for tracking movement (locomotion or directed motion) and the 
502 other for "activity quantitation", quantitation of general activity. In the directed motion assay,

503 CYP20A1 MO-injected larvae spent significantly more time at high speed $\left(\geq 0.5 \mathrm{~cm} . \mathrm{s}^{-1}\right)$ than

504 either non-injected or control MO-injected larvae, and also travelled more distance at high

505 speed (Fig. 7B). Hyperactivity or bursts of activity, evident as significantly longer periods of time

506 they spent at a high activity state, were typical of these larvae. This was reflected also in the

507 higher level of total activity, expressed as integrated values (Fig. 7C).

508

509 Discussion

510 In these studies, we cloned and sequenced the cDNA of CYP20A1 of the zebrafish TL

511 strain and found it identical to the zebrafish CYP20A1 coding sequence available in databases,

512 and with a high \% identity to the cloned human CYP20A1. Unusual features of the CYP20A1

513 sequence have been suggested before, based largely on inferences from genome sequences

514 (Sezutsu et al., 2013). The comparison of cloned zebrafish and human CYP20A1s establishes

515 that these unusual features are present in the expressed proteins from both species. Zebrafish

516 and human CYP20A1s have a highly conserved N-terminal motif that not only contains the

517 transmembrane domain typical of microsomal P450s, but also have an adjacent motif that could

518 possibly act as a signal peptide for mitochondrial targeting. This suggests that CYP20A1s might

519 have a bimodal distribution in the ER and mitochondria, similarly to some other vertebrate

520 P450s (Avadhani et al., 2011). Further work is needed to determine whether there is dual

521 subcellular localization of CYP20A1s.

522 The zebrafish CYP20A1 protein and the human ortholog contain a typical EXXR motif in

523 the K-helix, thought to be required for proper folding and heme inclusion (Sezutsu et al., 2013).

524 However, the I-helix and heme-binding regions were found to diverge significantly from the

525 classical P450 signature motifs. The absence of one residue at the beginning of the heme-

526 binding motif, and the Glu instead of a conserved Gly at the terminal portion, are unique to 
527 CYP20A1 when compared to other vertebrate P450s. Replacement of a non polar (Gly) by a 528 negatively charged (Glu) residue at the end of the heme-binding motif is intriguing considering

529 that the site where this change occurs is thought to be in close vicinity to the catalytic Cys in the 530 tertiary structure (Otyepka et al., 2007). The presence of a Gln residue at a central site of the

531 heme-binding motif, where usually an Arg or His residue is found, is also intriguing. The

532 signature Arg or His residue is responsible for the hydrogen bonding with one heme propionate

533 (Otyepka et al., 2007). It has been suggested that these hydrogen bonds not only can influence

534 the stability of hemeproteins, but also their reactivity and ligand selection (Ramos-Santana and

535 Lopez-Garriga, 2012). Note that GIn is also able to generate a hydrogen bond with the heme

536 propionate. These features imply there could be unusual catalytic properties of CYP20A1s, as

537 previously suggested (Sezutsu et al., 2013). In preliminary studies we failed to detect any

538 binding of cholesterol or arachidonic acid to recombinant zebrafish CYP20A1. Likewise, Stark et

al. (Stark et al., 2007) did not detect any activity of human CYP20A1 with cholesterol or

arachidonic acid or any of 9 other possible substrates, including neurotransmitters. However, in

a proteomic mapping of cholesterol-interacting proteins CYP20A1 was identified as possibly

interacting with trans-sterol (Hulce et al., 2013). The success at expressing recombinant

543 zebrafish CYP20A1 will facilitate the search for substrates, and could provide for engineering to

544 test possible functions.

$545 \quad$ The divergence observed in the I-helix, especially the absence of the conserved

546 threonine at the $5^{\text {th }}$ position of the motif, is similar to what is observed in zebrafish and human

547 CYP7s and CYP8s. These sequences differ from zebrafish CYP20A1 in having a Ser residue at

548 the second position of the motif in most cases (although a Ser is present in human CYP20A1), a

549 Gly residue at the fourth and an Asn residue at the fifth position. While the functional

550 significance of these I-helix features of CYP20A1 is unknown, it is notable that the presence of

551 an lle residue rather than Thr at the $5^{\text {th }}$ position of the I-helix motif also occurs in human

552 CYP5A1 (thromboxane synthase). Reverse-engineering experiments with human CYP5A1 have 
553 demonstrated a specific role for the lle residue in keeping rates of product formation by the

554 enzyme at low levels (Meling et al., 2015). The tight control of catalysis by CYP5A1 suggests

555 that the lle in CYP20A1 could perhaps act similarly to limit product formation by this enzyme.

556 Thus, too much or too little CYP20A1 activity could be detrimental.

557 Tissue distribution in reproductive, immune and nervous systems

558 The tissue distribution of CYP20A1 transcript in zebrafish was somewhat different from

559 that observed with rodents. Thus, in male mouse, abundant CYP20A1 expression was found in

560 spleen, followed by skeletal muscle, brain, heart, lung, kidney, testis and liver (Choudhary et al.,

561 2003). In male rat, CYP20A1 was most strongly expressed in liver, followed by lung, spleen,

562 kidney, heart, brain and testis (Takiguchi et al., 2010). Regardless of these differences, studies

563 with zebrafish, human (Stark et al., 2008) and rodents are consistent in pointing to relatively

564 high-level expression for CYP20A1 in reproductive, immune and nervous tissues. These

565 patterns are consistent with the putative TF binding sites in CYP20A1 proximal promoters.

566 The tissue distribution in zebrafish brain and eye also suggests a role for CYP20A1 in

567 development and maintenance and/or function of the nervous system, supporting the inference

568 from high level expression in human hippocampus, substantia nigra and retinal pigment

569 epithelium (Stark et al., 2008; Strunnikova et al., 2010). It also has been noted that traumatic

570 injury to both hippocampus and retinal pigment epithelium resulted in significant up-regulation of

571 CYP20A1 expression in these rodent tissues (Birnie et al., 2013; Hadziahmetovic et al., 2012).

572 Our observation that the optomotor response was slowed in the MO knockdown larvae is

573 consistent with some role in visual processes. Refining the localization of CYP20A1 expression

574 in zebrafish brain is an objective for further studies.

575 The data on CYP20A1 expression in the literature also reveal an enrichment of the

576 transcript in human neural progenitor cells derived from embryonic stem cells (Chan et al.,

577 2015) and expression, along with a limited set of other CYP genes, in human cord blood 
578 hematopoietic and early progenitor cells (Xu et al., 2014) These observations support a role for

579 CYP20A1 at early stages of cell differentiation in some tissues.

580 Response to agonists for nuclear and xenobiotic receptors

581 At micromolar doses, agonists for some nuclear receptors modestly, but significantly,

582 increased CYP20A1 transcript levels in zebrafish embryos. The responses conform to

583 predictions based on the presence of cis-response elements in the proximal promoter region of

584 CYP20A1s. In silico and in vivo studies point to regulation of CYP20A1 expression by nuclear

585 receptors involved in steroid response, cholesterol and lipid metabolism, and down-regulation

586 by the morphogen all-trans retinoic acid.

587 Among NR agonists, the highest increases of CYP20A1 expression were seen with

588 diethylstilbestrol (DES) and T0901317. In zebrafish, DES is a potent ER agonist (Gorelick and

589 Halpern, 2011). In mammals, it also acts as an inverse agonist for ERRs (Coward et al., 2001).

590 To our knowledge, there are no reports of antagonism of zebrafish ERRs by DES. However,

591 putative binding sites were specifically predicted for ERRa in proximal promoters of the

592 vertebrate CYP20A1s at a cut-off of $80 \%$ of the human core sequence identity. ERs exert

593 functions in reproduction and neuronal protection (Dahlman-Wright et al., 2006), while ERRs

594 control the diurnal expression of genes involved in fatty acid, cholesterol and bile acid

595 metabolism (Giguere et al., 2011).

596 An increase of CYP20A1 expression in zebrafish embryos also was observed with

597 T0901317 exposure. T0901317 is a benzenesulfonamide that is a potent agonist for LXR in

598 mammals, and also is an agonist for PXR and FXR (Houck et al., 2004; Mitro et al., 2007) and

599 an inverse agonist for RORa, and RORY (Kumar et al., 2010). T0901317 appears to be an

600 agonist for zebrafish LXR and FXR, but not PXR (Krasowski et al., 2011; Reschly et al., 2008).

601 In silico studies predicts the presence of binding sites for FXR and RORa in promoters of

602 vertebrate CYP20A1. A less stringent cut-off also identified binding sites for LXR (i.e., 70\%

603 similarity with human set of LXR binding sites). These observations suggest that a diversity of 
604 nuclear receptors could have been involved in the T0901317-mediated increase of CYP20A1

605 expression. The LXRs and FXR are involved in sterol and bile acid homeostasis (Kalaany and

606 Mangelsdorf, 2006), while RORs, which bind cholesterol, exert functions in brain and eye

607 development, and immunity (Jetten, 2009). In contrast, there was no effect on CYP20A1

608 expression by nanomolar doses of PCB 126, or 2,3,7,8-tetrachlorodibenzodioxin (data not

609 shown), pointing to a lack of involvement of the AHR. Immunoassay with antibodies to zebrafish

610 CYP20A1 would help to define localization of protein and responses to chemical treatment.

611 CYP20A1 knockdown effects

612 The potential involvement of CYP20A1 in neurobehavioral disorders is indicated not only

613 by the expression in zebrafish, rodent and human brain, but also by the effects of CYP20A1

614 knockdown investigated here. Zebrafish embryos in which CYP20A1 translation was partly

615 inhibited showed developmental delay at $24 \mathrm{hpf}$, and behavioral effects were evident at 1 and 5

$616 \mathrm{dpf}$. The latency in initiating an appropriate swimming response due to CYP20A1 knockdown in

617 the OMR assay can be described as a defective visuo-motor integration or coordination, or an

618 increased anxiety (Kalueff et al., 2014), rather than an impaired locomotory capacity. Larvae of

619 the CYP20A1 MO group also exhibited a substantial increase of activity and swimming speed

620 compared to the two control groups in the automated tracking-and-activity experiments. The

621 possibility that the confined settings of the multiwell plates elicited these phenotypes in the

622 CYP20A1 knockdown fish cannot be ruled out; thus, these bursts of activity and higher

623 swimming speeds could have been driven by anxiety (Maximino et al., 2010).

624 Intriguingly, these phenotypic effects of CYP20A1 knockdown in zebrafish are strongly

625 reminiscent of those seen in human patients diagnosed with microdeletions in the $2 q 33$

626 chromosome region where CYP20A1 is located. Patients with this rare syndrome variously

627 show psychomotor retardation, hyperactivity and bouts of anxiety, and a delayed visuomotor

628 coordination was specifically reported once (Balasubramanian et al., 2011; Tomaszewska et al.,

629 2013). Analyzing the specific regions deleted, which are displayed in Balasubramian et al. 
630 (Balasubramanian et al., 2011), reveals that the majority of patients (5-6 of 7) with deletions that

631 included CYP20A1 presented with hyperactivity, while this phenotype was rarely observed in

632 patients with deletions that excluded CYP20A1 (1-3 of 13). The present results with knockdown

633 of CYP20A1 in zebrafish, and the 2q33 microdeletion patients, suggests that this orphan P450

634 could well be involved in neurobehavioral hyperactivity.

635 Response to methylmercury

636 The possible effects of a neurotoxicant on CYP20A1 expression were assessed by

637 exposing zebrafish embryos to $\mathrm{MeHg}$, among the chemicals suggested to contribute to

638 hyperactivity disorder (Grandjean and Landrigan, 2014). These experiments revealed a dose-

639 dependent reduction of CYP20A1 transcript abundance following $24 \mathrm{~h}$ exposure of zebrafish,

640 beginning at $24 \mathrm{hpf}$. The higher dose tested resulted in the strongest decline of CYP20A1

641 expression. Notably, the MeHg concentration threshold in human cord blood that is associated

642 with the detection of behaviors typical of attention deficit hyperactivity disorder (ADHD) during

643 childhood is very close to the lowest dose we tested (Boucher et al., 2012). Providing that this

644 effect on CYP20A1 expression also occurs in human and translates into changes at the protein

645 level, it is tempting to envisage a link between prenatal exposure to MeHg, CYP20A1 repression

646 (with detrimental developmental consequences) and the onset of ADHD. We are investigating

647 further the mechanism of the MeHg effect on CYP20A1, and how CYP20A1 may function in

648 neurological processes. Such studies will include direct comparison of behavioral change and

649 CYP20A1 expression following MeHg exposures, as well as CYP20A1 responses to other

650 neurotoxicants.

651

652 Conclusion

653 In this study, we cloned and examined properties of the zebrafish orphan P450 CYP20A1.

654 Peculiar structural features of zebrafish, human and other vertebrate CYP20A1s are clues to an 
655 unusual catalytic function. The expression patterns of CYP20A1 transcript, and putative

656 transcription factor binding sites suggest potential involvement in reproductive, immune and

657 neuronal functions, as well as steroid response, lipid and cholesterol metabolism. A possible

658 involvement in neuronal processes is strongly indicated by the behavioral effects occurring in

659 zebrafish larvae treated with a morpholino against CYP20A1. The effects of knockdown -

660 especially hyperactivity - are reminiscent of those in humans who have a microdeletion that

661 includes loss of CYP20A1 gene. The MeHg-mediated reduction of CYP20A1 transcript levels in

662 zebrafish embryos points to the possibility that disruption of CYP20A1 expression may be

663 involved in some neurobehavioral disorders. Studies of CYP20A1 expression with additional

664 toxicants are underway. Future studies will investigate whether over-expression can reverse

665 behavioral deficits seen with $\mathrm{MO}$ treatments or $\mathrm{MeHg}$, or whether overexpression also could be

666 detrimental, which could occur if tight regulation of the enzyme might be necessary. The results

667 presented here together shed new light on the possible biological and toxicological significance

668 of the orphan CYP20A1 that has thus far resisted "deorphanization". 


\section{Acknowledgements}

670 This work was supported in part by NIH Superfund Research Program at Boston University,

671 grant P42ES007381 from the National Institute of Environmental Health Sciences (NIEHS) (JJS,

672 JVG, AK and BL), by a Belgian-American Educational Foundation (BAEF) Postdoctoral

673 Fellowship (BL), the Japan Society for the Promotion of Science Postdoctoral Fellowship and

674 Postdoctoral Fellowship for Research Abroad to AK (nos. 4313 and 820, respectively), and

675 NIEHS grant P30ES000210. Additional support (JJS) was provided by NIH grant P01ES021923

676 and National Science Foundation Grant OCE-1314642 through the Woods Hole Center for

677 Oceans and Human Health, and from the Aquastress project funded by the BELSPO; Belgian

678 Federal Science Policy Office, P7/31. We gratefully acknowledge the assistance of Matthew

679 Takata for preparation of RNA from tissue samples, Sibel Karchner and Diana Franks for help

680 with TnT assay, Rachel Harbeitner for assistance with qPCR, and Victoria Starczak for help with 681 statistics.

682

683 


\section{References}

Avadhani, N.G., Sangar, M.C., Bansal, S., Bajpai, P., 2011. Bimodal targeting of cytochrome P450s to endoplasmic reticulum and mitochondria: the concept of chimeric signals. Febs $J$ 278, 4218-4229.

Bairoch, A., Apweiler, R., Wu, C.H., Barker, W.C., Boeckmann, B., Ferro, S., Gasteiger, E., Huang, H., Lopez, R., Magrane, M., Martin, M.J., Natale, D.A., O'Donovan, C., Redaschi, N., Yeh, L.S., 2005. The Universal Protein Resource (UniProt). Nucleic Acids Res 33, D154159.

Balasubramanian, M., Smith, K., Basel-Vanagaite, L., Feingold, M.F., Brock, P., Gowans, G.C., Vasudevan, P.C., Cresswell, L., Taylor, E.J., Harris, C.J., Friedman, N., Moran, R., Feret, H., Zackai, E.H., Theisen, A., Rosenfeld, J.A., Parker, M.J., 2011. Case series: 2q33.1 microdeletion syndrome--further delineation of the phenotype. J Med Genet 48, 290-298.

Barnes, H.J., Arlotto, M.P., Waterman, M.R., 1991. Expression and enzymatic activity of recombinant cytochrome P450 17 alpha-hydroxylase in Escherichia coli. Proceedings of the National Academy of Sciences of the United States of America 88, 5597-5601.

Birnie, M., Morrison, R., Camara, R., Strauss, K.I., 2013. Temporal changes of cytochrome P450 (Суp) and eicosanoid-related gene expression in the rat brain after traumatic brain injury. BMC Genomics 14, 303.

Boucher, O., Jacobson, S.W., Plusquellec, P., Dewailly, E., Ayotte, P., Forget-Dubois, N., Jacobson, J.L., Muckle, G., 2012. Prenatal methylmercury, postnatal lead exposure, and evidence of attention deficit/hyperactivity disorder among Inuit children in Arctic Quebec. Environmental health perspectives 120, 1456-1461.

Bryson, K., McGuffin, L.J., Marsden, R.L., Ward, J.J., Sodhi, J.S., Jones, D.T., 2005. Protein structure prediction servers at University College London. Nucleic Acids Res 33, W36-38.

Cartharius, K., Frech, K., Grote, K., Klocke, B., Haltmeier, M., Klingenhoff, A., Frisch, M.,

713 2015. Transcriptional profiling of MEF-2 regulated genes in human neural progenitor cells derived from embryonic stem cells. Genomics Data 3, 24-27.

715 Cheng, J., Baldi, P., 2005. Three-stage prediction of protein beta-sheets by neural networks, 716 alignments and graph algorithms. Bioinformatics 21 Suppl 1, i75-84. 
717 Choudhary, D., Jansson, I., Schenkman, J.B., Sarfarazi, M., Stoilov, I., 2003. Comparative

718 expression profiling of 40 mouse cytochrome P450 genes in embryonic and adult tissues.

719 Archives of biochemistry and biophysics 414, 91-100.

720 Coward, P., Lee, D., Hull, M.V., Lehmann, J.M., 2001. 4-Hydroxytamoxifen binds to and

721 deactivates the estrogen-related receptor gamma. Proceedings of the National Academy of

722 Sciences of the United States of America 98, 8880-8884.

723 Dahlman-Wright, K., Cavailles, V., Fuqua, S.A., Jordan, V.C., Katzenellenbogen, J.A., Korach,

724 K.S., Maggi, A., Muramatsu, M., Parker, M.G., Gustafsson, J.A., 2006. International Union of

725 Pharmacology. LXIV. Estrogen receptors. Pharmacol Rev 58, 773-781.

726 Flicek, P., Ahmed, I., Amode, M.R., Barrell, D., Beal, K., Brent, S., Carvalho-Silva, D., Clapham,

727 P., Coates, G., Fairley, S., Fitzgerald, S., Gil, L., Garcia-Giron, C., Gordon, L., Hourlier, T.,

728 Hunt, S., Juettemann, T., Kahari, A.K., Keenan, S., Komorowska, M., Kulesha, E., Longden,

729 I., Maurel, T., McLaren, W.M., Muffato, M., Nag, R., Overduin, B., Pignatelli, M., Pritchard,

730 B., Pritchard, E., Riat, H.S., Ritchie, G.R., Ruffier, M., Schuster, M., Sheppard, D., Sobral,

731 D., Taylor, K., Thormann, A., Trevanion, S., White, S., Wilder, S.P., Aken, B.L., Birney, E.,

732 Cunningham, F., Dunham, I., Harrow, J., Herrero, J., Hubbard, T.J., Johnson, N., Kinsella,

733 R., Parker, A., Spudich, G., Yates, A., Zadissa, A., Searle, S.M., 2013. Ensembl 2013.

$734 \quad$ Nucleic Acids Res 41, D48-55.

735 Giguere, V., Dufour, C.R., Eichner, L.J., Deblois, G., Cermakian, N., 2011. Estrogen-related

736 receptor alpha, the molecular clock, and transcriptional control of metabolic outputs. Cold

737 Spring Harb Symp Quant Biol 76, 57-61.

738 Goldstone, J.V., McArthur, A.G., Kubota, A., Zanette, J., Parente, T., Jonsson, M.E., Nelson,

739 D.R., Stegeman, J.J., 2010. Identification and developmental expression of the full

740 complement of Cytochrome P450 genes in Zebrafish. BMC Genomics 11, 643.

741 Gorelick, D.A., Halpern, M.E., 2011. Visualization of estrogen receptor transcriptional activation

742 in zebrafish. Endocrinology 152, 2690-2703.

743 Grandjean, P., Landrigan, P.J., 2014. Neurobehavioural effects of developmental toxicity.

$744 \quad$ Lancet Neurol 13, 330-338.

745 Gupta, T., Mullins, M.C., 2010. Dissection of organs from the adult zebrafish. J Vis Exp.

746 Hadziahmetovic, M., Kumar, U., Song, Y., Grieco, S., Song, D., Li, Y., Tobias, J.W., Dunaief,

747 J.L., 2012. Microarray analysis of murine retinal light damage reveals changes in iron

748 regulatory, complement, and antioxidant genes in the neurosensory retina and isolated

749 RPE. Invest Ophthalmol Vis Sci 53, 5231-5241. 
Hassan, S.A., Moussa, E.A., Abbott, L.C., 2012. The effect of methylmercury exposure on early central nervous system development in the zebrafish (Danio rerio) embryo. J Appl Toxicol 32, 707-713. Honkakoski, P., Negishi, M., 2000. Regulation of cytochrome P450 (CYP) genes by nuclear receptors. The Biochemical journal 347, 321-337. Houck, K.A., Borchert, K.M., Hepler, C.D., Thomas, J.S., Bramlett, K.S., Michael, L.F., Burris, T.P., 2004. T0901317 is a dual LXR/FXR agonist. Mol Genet Metab 83, 184-187.

\section{Hulce, J.J., Cognetta, A.B., Niphakis, M.J., Tully, S.E., Cravatt, B.F., 2013. Proteome-wide} mapping of cholesterol-interacting proteins in mammalian cells. Nat Methods 10, 259-264. James-Zorn, C., Ponferrada, V.G., Jarabek, C.J., Burns, K.A., Segerdell, E.J., Lee, J., Snyder, K., Bhattacharyya, B., Karpinka, J.B., Fortriede, J., Bowes, J.B., Zorn, A.M., Vize, P.D., 2013. Xenbase: expansion and updates of the Xenopus model organism database. Nucleic Acids Res 41, D865-870.

Jetten, A.M., 2009. Retinoid-related orphan receptors (RORs): critical roles in development, immunity, circadian rhythm, and cellular metabolism. Nucl Recept Signal 7, e003. Jonsson, M.E., Jenny, M.J., Woodin, B.R., Hahn, M.E., Stegeman, J.J., 2007a. Role of AHR2 in the expression of novel cytochrome P450 1 family genes, cell cycle genes, and morphological defects in developing zebra fish exposed to 3,3',4,4',5-pentachlorobiphenyl or 2,3,7,8-tetrachlorodibenzo-p-dioxin. Toxicological sciences : an official journal of the Society of Toxicology 100, 180-193.

Jonsson, M.E., Orrego, R., Woodin, B.R., Goldstone, J.V., Stegeman, J.J., 2007b. Basal and 3,3',4,4',5-pentachlorobiphenyl-induced expression of cytochrome P450 1A, 1B and 1C genes in zebrafish. Toxicology and applied pharmacology 221, 29-41.

Kalaany, N.Y., Mangelsdorf, D.J., 2006. LXRS and FXR: the yin and yang of cholesterol and fat metabolism. Annu Rev Physiol 68, 159-191.

Kalueff, A.V., Stewart, A.M., Gerlai, R., 2014. Zebrafish as an emerging model for studying complex brain disorders. Trends in pharmacological sciences 35, 63-75.

Krasowski, M.D., Ai, N., Hagey, L.R., Kollitz, E.M., Kullman, S.W., Reschly, E.J., Ekins, S., green-spotted pufferfish (Tetraodon nigriviridis) and other non-mammalian species. BMC Biochem 12, 5.

Krogh, A., Larsson, B., von Heijne, G., Sonnhammer, E.L., 2001. Predicting transmembrane protein topology with a hidden Markov model: application to complete genomes. J Mol Biol 305, 567-580. 
784 Kubota, A., Bainy, A.C., Woodin, B.R., Goldstone, J.V., Stegeman, J.J., 2013. The cytochrome P450 2AA gene cluster in zebrafish (Danio rerio): expression of CYP2AA1 and CYP2AA2 and response to phenobarbital-type inducers. Toxicology and applied pharmacology 272 , 172-179.

Kumar, N., Solt, L.A., Conkright, J.J., Wang, Y., Istrate, M.A., Busby, S.A., Garcia-Ordonez,

Lamb, D.C., Waterman, M.R., 2013. Unusual properties of the cytochrome P450 superfamily. Philos Trans R Soc Lond B Biol Sci 368, 20120434.

Livak, K.J., Schmittgen, T.D., 2001. Analysis of relative gene expression data using real-time quantitative PCR and the 2(-Delta Delta C(T)) Method. Methods 25, 402-408.

Maaswinkel, H., Li, L., 2003. Spatio-temporal frequency characteristics of the optomotor response in zebrafish. Vision Res 43, 21-30.

Maximino, C., de Brito, T.M., da Silva Batista, A.W., Herculano, A.M., Morato, S., Gouveia, A., Jr., 2010. Measuring anxiety in zebrafish: a critical review. Behav Brain Res 214, 157-171.

Meling, D.D., Zelasko, S., Kambalyal, A., Roy, J., Das, A., 2015. Functional role of the conserved i-helix residue 1346 in CYP5A1-Nanodiscs. Biophys Chem 200-201, 34-40.

Mitro, N., Vargas, L., Romeo, R., Koder, A., Saez, E., 2007. T0901317 is a potent PXR ligand: implications for the biology ascribed to LXR. FEBS letters 581, 1721-1726.

Morrison, A.M., Goldstone, J.V., Lamb, D.C., Kubota, A., Lemaire, B., Stegeman, J.J., 2014. Identification, modeling and ligand affinity of early deuterostome CYP51s, and functional characterization of recombinant zebrafish sterol 14alpha-demethylase. Biochimica et biophysica acta 1840, 1825-1836.

Nakai, K., Horton, P., 1999. PSORT: a program for detecting sorting signals in proteins and predicting their subcellular localization. Trends Biochem Sci 24, 34-36.

812 Nelson, D.R., Goldstone, J.V., Stegeman, J.J., 2013. The cytochrome P450 genesis locus: the 813 origin and evolution of animal cytochrome P450s. Philos Trans R Soc Lond B Biol Sci 368, 81420120474.

815 Otyepka, M., Skopalik, J., Anzenbacherova, E., Anzenbacher, P., 2007. What common 816 structural features and variations of mammalian P450s are known to date? Biochimica et 817 biophysica acta 1770, 376-389. 
818 Petersen, B., Petersen, T.N., Andersen, P., Nielsen, M., Lundegaard, C., 2009. A generic

819 method for assignment of reliability scores applied to solvent accessibility predictions. BMC

$820 \quad$ Struct Biol 9, 51.

821 Petersen, T.N., Brunak, S., von Heijne, G., Nielsen, H., 2011. SignalP 4.0: discriminating signal

822 peptides from transmembrane regions. Nat Methods 8, 785-786.

823 Ramos-Santana, B.J., Lopez-Garriga, J., 2012. Tyrosine B10 triggers a heme propionate

824 hydrogen bonding network loop with glutamine E7 moiety. Biochemical and biophysical

825 research communications $424,771-776$.

826 Reschly, E.J., Ai, N., Welsh, W.J., Ekins, S., Hagey, L.R., Krasowski, M.D., 2008. Ligand

827 specificity and evolution of liver X receptors. J Steroid Biochem Mol Biol 110, 83-94.

828 Rost, B., Yachdav, G., Liu, J., 2004. The PredictProtein server. Nucleic Acids Res 32, W321-

829326.

830 Sezutsu, H., Le Goff, G., Feyereisen, R., 2013. Origins of P450 diversity. Philos Trans R Soc

$831 \quad$ Lond B Biol Sci 368, 20120428.

832 Stark, K., Guengerich, F.P., 2007. Characterization of orphan human cytochromes P450. Drug

833 Metab Rev 39, 627-637.

834 Stark, K., Wu, Z.L., Bartleson, C.J., Guengerich, F.P., 2008. mRNA distribution and

835 heterologous expression of orphan cytochrome P450 20A1. Drug Metab Dispos 36, 1930-

8361937.

837 Strunnikova, N.V., Maminishkis, A., Barb, J.J., Wang, F., Zhi, C., Sergeev, Y., Chen, W.,

838 Edwards, A.O., Stambolian, D., Abecasis, G., Swaroop, A., Munson, P.J., Miller, S.S., 2010.

839 Transcriptome analysis and molecular signature of human retinal pigment epithelium.

$840 \quad$ Human molecular genetics 19, 2468-2486.

841 Takiguchi, M., Darwish, W.S., Ikenaka, Y., Ohno, M., Ishizuka, M., 2010. Metabolic activation of

842 heterocyclic amines and expression of CYP1A1 in the tongue. Toxicological sciences : an

843 official journal of the Society of Toxicology 116, 79-91.

844 Thisse, B., Thisse, C., 2004. Fast release clones: a high throughput expression analysis. ZFIN

845 direct data submision (http://zfin.org).

846 Tomaszewska, A., Podbiol-Palenta, A., Boter, M., Geisler, G., Wawrzkiewicz-Witkowska, A.,

847 Galjaard, R.J., Zajaczek, S., Srebniak, M.I., 2013. Deletion of 14.7 Mb 2q32.3q33.3 with a

848 marfanoid phenotype and hypothyroidism. Am J Med Genet A 161, 2347-2351.

849 Truong, L., Reif, D.M., St Mary, L., Geier, M.C., Truong, H.D., Tanguay, R.L., 2014.

850 Multidimensional in vivo hazard assessment using zebrafish. Toxicological sciences : an

851 official journal of the Society of Toxicology 137, 212-233. 
852 Werck-Reichhart, D., Feyereisen, R., 2000. Cytochromes P450: a success story. Genome Biol 8531 , REVIEWS3003.

854 Wirdefeldt, K., Adami, H.O., Cole, P., Trichopoulos, D., Mandel, J., 2011. Epidemiology and 855 etiology of Parkinson's disease: a review of the evidence. Eur J Epidemiol 26 Suppl 1, S185658.

857 Xu, S., Ren, Z., Wang, Y., Ding, X., Jiang, Y., 2014. Preferential expression of cytochrome CYP $858 \quad$ CYP2R1 but not CYP1B1 in human cord blood hematopoietic stem and progenitor cells.

863 Zhou, Y., Dougherty, J.H., Jr., Hubner, K.F., Bai, B., Cannon, R.L., Hutson, R.K., 2008.

864 Abnormal connectivity in the posterior cingulate and hippocampus in early Alzheimer's 865 disease and mild cognitive impairment. Alzheimers Dement 4, 265-270.

866

867

868 
868 Figure legends

869

870

871

872

Figure 1. Alignment of zebrafish and human CYP20A1 proteins: Locations of P450

873 signature motifs and predicted secondary structure are illustrated. Yellow boxes identify a-

874 helices and blue boxes $\beta$-strands. Green line locates the position of the predicted

875 transmembrane region, and dashed purple line that of a putative signaling peptide suggested to 876 target the protein to mitochondria.

877

878 Figure 2. Reduced, CO-difference spectrum of heterologously expressed zebrafish

879 CYP20A1: Zebrafish CYP20A1 was expressed in E. coli cells after $\mathrm{N}$-terminal modification. The 880 reduced difference spectrum shows a peak at $450 \mathrm{~nm}$, as expected, as well as a peak at $420 \mathrm{~nm}$

881 indicating partial loss of the cysteine thiolate linkage to the heme, possibly due to the non-native 882 protein expression in E coli.

883

884 Figure 3. Expression of CYP20A1 transcript in zebrafish tissues: For both sexes, transcript 885 levels were measured by real-time quantitative PCR (qPCR) using a double reference gene 886 approach (elongation factor $1 \alpha$ and aryl hydrocarbon receptor nuclear translocator 2), and data

887 are expressed relative (i.e., fold change) to transcript content in liver. Pools of organs were 888 prepared and qPCR was run on three pools each for females (Figure 3A) and males (Figure 889 3B). In the second set, qPCR was run on three pools from males only (Figure 3C). Data are 890 presented as mean \pm SD. Different letters indicate significant difference of expression in organs 891 of males and females separately (Kruskal-Wallis ANOVA with post-hoc Z-adjusted Mann892 Whitney U test).

893 
894 Figure 4. Expression of CYP20A1 in zebrafish embryos exposed to agonists for nuclear

895 receptors: (a) Responses to diethylstilbestrol (black), androstenedione (white), and

896 dexamethasone (grey). (b) Responses to T0901317 (black), farnesol isomers (white), and 5-

897 pregnen-3ß-ol-20-one (grey). Transcript levels were measured by real-time quantitative PCR

898 (qPCR) using a double reference gene approach (elongation factor 1 $\alpha$ and aryl hydrocarbon

899 receptor nuclear translocator 2). Exposures were from 24 to 72 hours post-fertilization on pools

900 of 15 embryos in $60 \mathrm{ml}$ of $0.3 \times$ Danieau's solution with $0.1 \%$ of dimethylsulfoxide as carrier.

901 Results were expressed as fold change from results obtained with carrier alone. Data are

902 presented as mean \pm SD of 4 replicates. Since the various doses of each chemical were tested

903 in separate experiments, analyses were conducted with a 2-tailed Student's t-test. Asterisks

904 indicate levels of statistical significance ( ${ }^{*}$ for $p \leq 0.05 ;{ }^{* *}$ for $\left.p \leq 0.01\right)$.

905

906 Figure 5. Expression of CYP20A1 in zebrafish embryos exposed to methylmercury

907 chloride: Transcript levels were measured by real-time quantitative PCR (qPCR) using a

908 double reference gene approach (elongation factor $1 \alpha$ and aryl hydrocarbon receptor nuclear

909 translocator 2). Exposures were from 24 to 48 hours post-fertilization on pools of 15 embryos in

$91060 \mathrm{ml}$ of $1 \mathrm{X}$ E3 medium with $0.1 \%$ of distilled water as carrier. Results are expressed relative

911 (i.e., fold change) to those obtained with carrier alone. Data are presented as mean \pm SD of 4

912 replicates. Analysis of the difference in CYP20A1 expression from solvent controls was

913 conducted with one-way ANOVA followed by post hoc LSD Fisher's test. Asterisks indicate

914 levels of statistical significance $\left({ }^{*}\right.$ for $p \leq 0.05 ;{ }^{* *}$ for $\left.p \leq 0.01\right)$.

915

916 Figure 6. In ovo tail flexion movement response in zebrafish embryos treated with

917 CYP20A1 morpholino: In ovo tail flexion movement was automatically analyzed at 24 hours

918 post-fertilization in zebrafish embryos of the Tropical 5D wild-type strain after injection with

919 CYP20A1 morpholino (MO A) (top line) or control morpholino (MO B). Embryos that were not 
injected (Uninj) were also used in the blind test. Individuals were placed one per well of a 96well plate. The red vertical lines indicate the time of a single bright, visible flashlight. The Y-axis values are an index of tail flexion (i.e., pixel changes between successive frames), and the Xaxis values are given in seconds. Asterisks and labels indicate the statistically significant hyperactivity associated with CYP20A1 morpholino in both the baseline (dark, un-stimulated) phase and in the first excitatory period (i.e., following first pulse of bright light), and with control morpholino in just the first excitatory period.

\section{Figure 7. Optomotor and locomotory effects of CYP20A1 knockdown in zebrafish larvae:}

TL embryos were injected at the two-to-four cell stage with a morpholino (MO) specific for CYP20A1 or a control morpholino (Ctrl MO). Batch-specific embryos that were not injected were also used (Non injected). Assays were conducted on 5 day-old fish. In the optomotor response study (OMR; Figure 7A), a sine wave of black and white stripes $(6.5 \mathrm{~cm}$ periodicity; $0.5 \mathrm{~Hz})$ was tested (20 larvae per replicate per group; tested individually) and the sum of the straight distances travelled was used to compare OMR between groups every $15 \mathrm{~s}$, up to $1 \mathrm{~min}$. Upper and lower panels show results from the first time bins as percentages of the straight distance travelled relative to non-injected larvae. The automated ViewPoint Zebrabox® was used to track locomotion (Figure 7B) and activity (Figure 7C) of the larvae in 24-well plates under constant illumination. 8 larvae from each group were simultaneously tested in consecutive 10-minute movie sessions (one for movement tracking and one for activity quantitation). For movement tracking, thresholds were: $0.5 \mathrm{~cm} . \mathrm{s}^{-1}$ for large and 0.1 for no movement. Upper and lower panels of Figure 7B respectively depict the time and distance travelled by larvae at high speed (i.e., $\geq$ $0.5 \mathrm{~cm} . \mathrm{s}^{-1}$ ). For activity quantitation, thresholds were: 10 pixels per frame for burst and one for freeze. Upper and lower panels of Figure $7 \mathrm{C}$ respectively depict the time spent by larvae in a hyperactive state (i.e., $\geq 10$ pixels per frame) and their total level of activity (i.e., area under

945 curve). Data are presented as mean \pm SEM of six replicates for OMR and three replicates for 
946 tracking and activity, and were analyzed with Kruskal-Wallis ANOVA followed by Z-adjusted

947 Mann-Whitney U test (OMR) or one-way ANOVA followed by post hoc LSD Fisher's test

948 (tracking-and-activity). Differences of CYP20A1 MO groups from non-injected controls are

949 indicated by asterisks, $\left({ }^{*} \mathrm{p} \leq 0.05 ;{ }^{* *} \mathrm{p} \leq 0.01\right)$ and from Ctrl MO by diamonds $(\bullet \mathrm{p} \leq 0.05 ; \bullet p$

$950 \leq 0.01)$.

951

952

953 
Table 1. P450 signature motifs in CYP20A1 and other selected CYPs in zebrafish.

\begin{tabular}{|c|c|c|c|}
\hline $\begin{array}{c}\text { P450 } \\
\text { isoform }\end{array}$ & $\begin{array}{l}\text { I helix motif } \\
\text { [G/AGXE/DTX] }\end{array}$ & $\begin{array}{c}\text { K helix motif } \\
{[\mathrm{EXXR}]}\end{array}$ & $\begin{array}{l}\text { Heme binding motif } \\
\text { [FXXGXR/HXCXG] }\end{array}$ \\
\hline CYP20A1 & AGCVIT & ETVR & F-SGSQACPE \\
\hline CYP1A & AGFDTI & EIFR & FGLGKRRCIG \\
\hline CYP2AA2 & AGTDTT & EIQR & FSLGPRACLG \\
\hline CYP3C1 & GGYETT & ESMR & FGLGPRNCIG \\
\hline CYP5A1 & AGYETS & ESLR & FGAGPRSCVG \\
\hline CYP7A1 & A $\underline{S} Q \underline{G N T}$ & EAMR & FGSGV $\underline{T}$ KCPG \\
\hline CYP7C1 & A $\underline{S V G N T}$ & ESLR & FGSGATQCPG \\
\hline CYP8A1 & VTQGNA & ETLR & $\underline{\text { WGTEDNLCPG }}$ \\
\hline CYP8B1 & A $\underline{S Q G N T}$ & ETLR & $\underline{\text { WGAGTTMCPG }}$ \\
\hline CYP8B2 & A $\underline{S} Q \underline{G N T}$ & ETLR & $\underline{\text { WGAGTTMCPG }}$ \\
\hline CYP8B3 & A $\underline{S} Q \underline{G N T}$ & ETLR & WGAGTTMCPG \\
\hline CYP11C1 & GGVDTT & ETLR & FGFGSRQCVG \\
\hline CYP39A1 & A $\underline{S} L \underline{A N A}$ & EAIR & FGGGKNQNQPG \\
\hline CYP51 & AGQHTS & ETLR & FGAGRHRCIG \\
\hline
\end{tabular}

Underlined residues in some CYP isoforms (including CYP20A1) are those differing from the classical P450 signature motif reported in the heading of each column. See sources in Table S6. 
Table 2. Recurring putative binding sites for families of nuclear receptors in proximal promoters of CYP20A1s in vertebrates.

\begin{tabular}{|lccccc|}
\hline \multicolumn{1}{|c}{ Matrix family } & $\begin{array}{c}\text { Danio } \\
\text { rerio }\end{array}$ & $\begin{array}{c}\text { Xenopus } \\
\text { tropicalis }\end{array}$ & $\begin{array}{c}\text { Gallus } \\
\text { gallus }\end{array}$ & $\begin{array}{c}\text { Rattus } \\
\text { norvegicus }\end{array}$ & $\begin{array}{c}\text { Homo } \\
\text { sapiens }\end{array}$ \\
\hline $\begin{array}{l}\text { Steroidogenic Factor } \\
\text { (V\$SF1F) }\end{array}$ & 2 & 11 & 9 & 6 & 6 \\
$\begin{array}{l}\text { Estrogen Response } \\
\text { (V\$EREF) }\end{array}$ & 3 & 5 & 8 & 7 & 2 \\
$\begin{array}{l}\text { V-ERB and RAR- } \\
\text { related Orphan } \\
\text { Receptor Alpha }\end{array}$ & 3 & 5 & 5 & 7 & 13 \\
$\begin{array}{l}\text { (VRORA) } \\
\text { Peroxisome }\end{array}$ & & & & & \\
$\begin{array}{l}\text { Proliferator-activated } \\
\text { Receptor (V\$PERO) }\end{array}$ & 6 & 22 & 14 & 9 & 13 \\
$\begin{array}{l}\text { Glucocorticoid } \\
\text { Response and }\end{array}$ & 8 & 12 & 14 & 14 & 7 \\
Related (V\$REF) & & & & & \\
\hline
\end{tabular}




\section{FIGURES}

Transmembrane region Mito signal?

D. rerio MLDFA IFAVTFVIILI GAVLYLYPSSRRASGVPGLNPTEEKDGNLQD IVNKGSLHEFLVGLHDEFGSVASFWFGARPVVS

H. sapiens MLDFAIFAVTFLLALVGAVLYLYPASRQAAGI PGITPTEEKDGNLPDIVNSGSLHEFLVNLHERY GPVV SFWFGRRLVVS

$\begin{array}{llllllll}90 & 100 & 110 & 120 & 130 & 140 & 150 & 160\end{array}$

D. rerio LGAVNQLRQH INPNWT TDSFETMLKSLLGYQSGSGV GLTESMMRKKVYEGA I NKT LENNFPLLLQQVEELVDKWASY PKS H. sapiens LGTVDVLKQH INPNKT SDPFETMLKSLLRYQSGGGS V VENHMRKKLY ENGVTDSLKSNFALLLKLSEELLDKWLSYPET

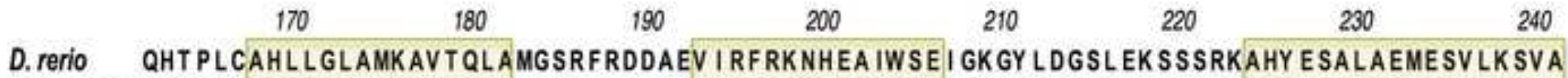
H. sapiens QHVPLSQHMLGFAMKSVTQMVMGSTFEDDQEV IRFQKNHGTVWSEI GKGFLDGSLDKNMT RKKQY EDALMQLESVLRNII

$250 \quad 260 \quad 270 \quad \begin{array}{llllll}280 & 290 & 300 & 310 & 320\end{array}$

D. rerio KQRPGQGSSQ-SFVNY LLQANLTERQVMEDGMV FT LAGCV ITANLC IWAVHFLSV SEAVQDRLYHELVEV LGDEPV SLEK H. sapiens KERKGRNF SQH IFIDSLVQGNLNDQQI LEDSMI FSLASCIITIAKLCTWAICFLTT SEEV QKKLYEEINQV FGNGPVTPEK

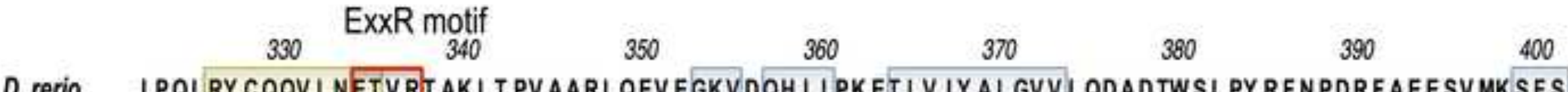

D. rerio I PQLRY CQQV LNETVRTAKLTPVAARLQEVEGKVDQHI I PKETLVIYALGVVLQDADTWSLPYRFNPDRFAEESVMKSFS

Heme binding motif

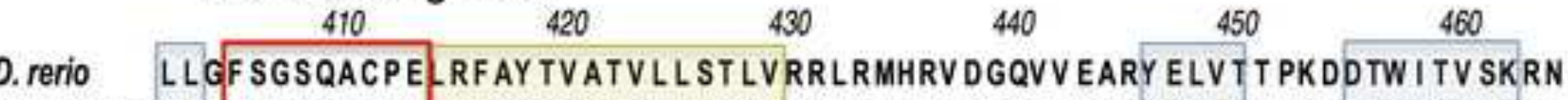

H. sapiens SLGESGT QECPE RFAYMVTTVLLSVLVKRLHLLSVEGQV I ETKYELVT SSREEAWITV SKRY 


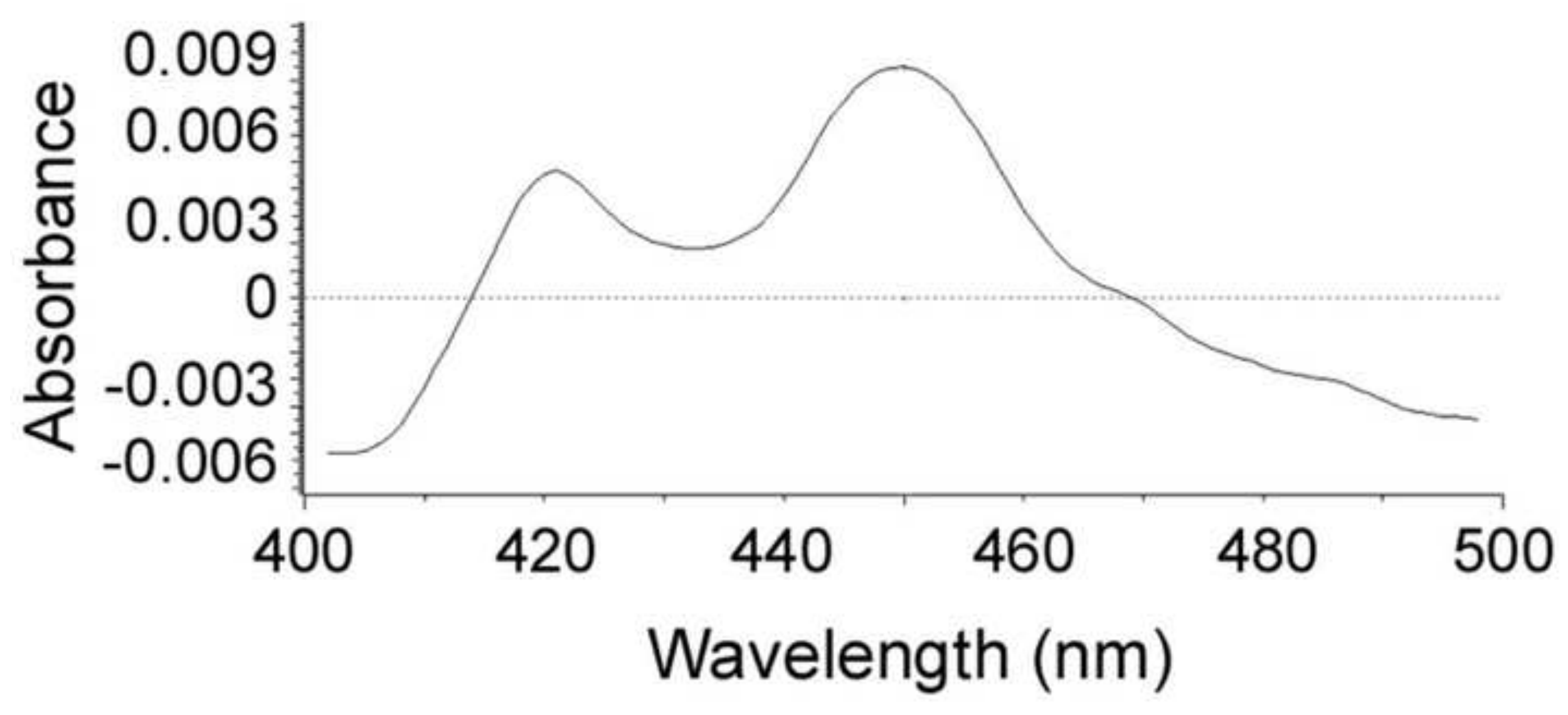


Figure $3 \mathrm{~A}$
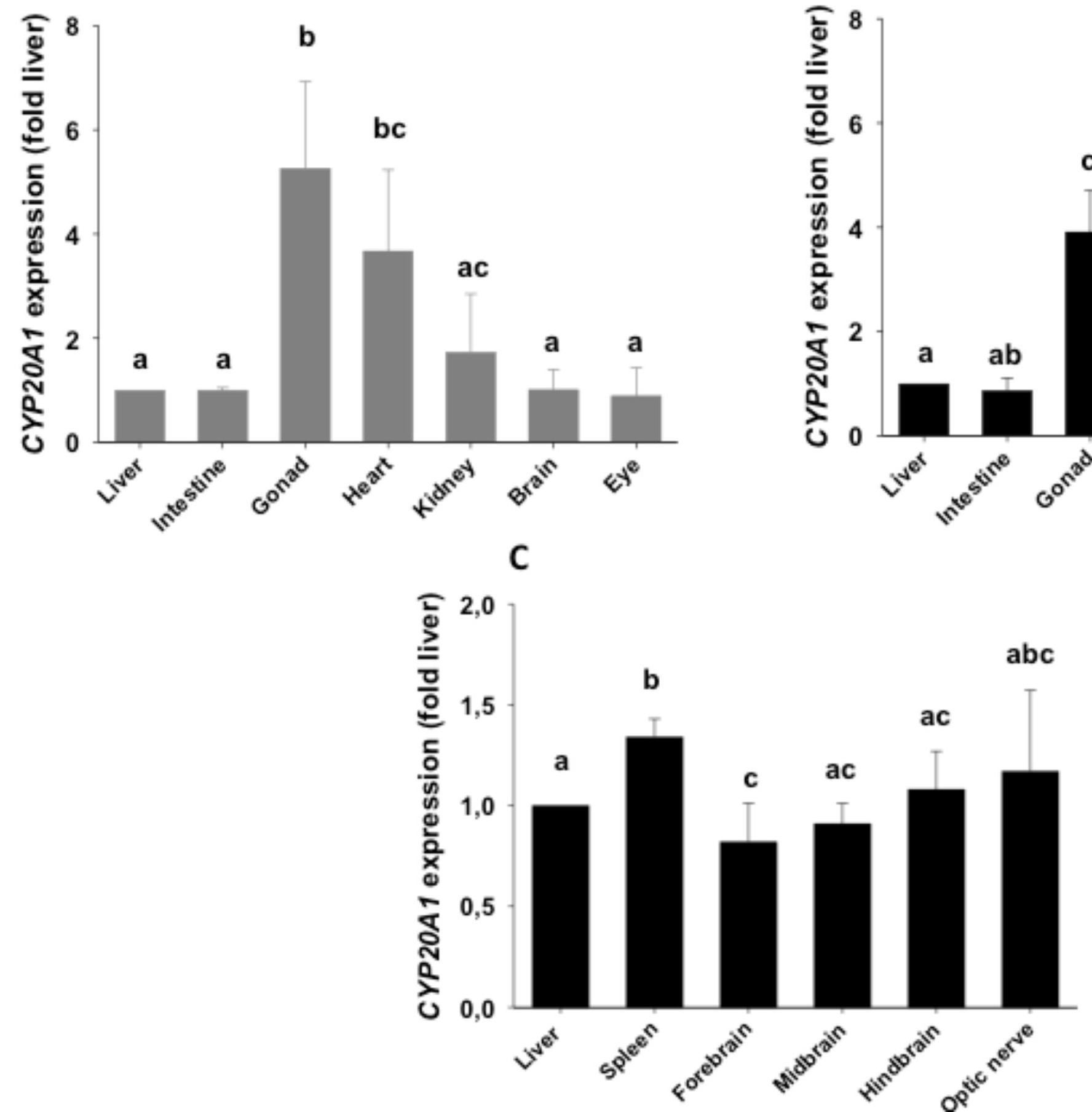

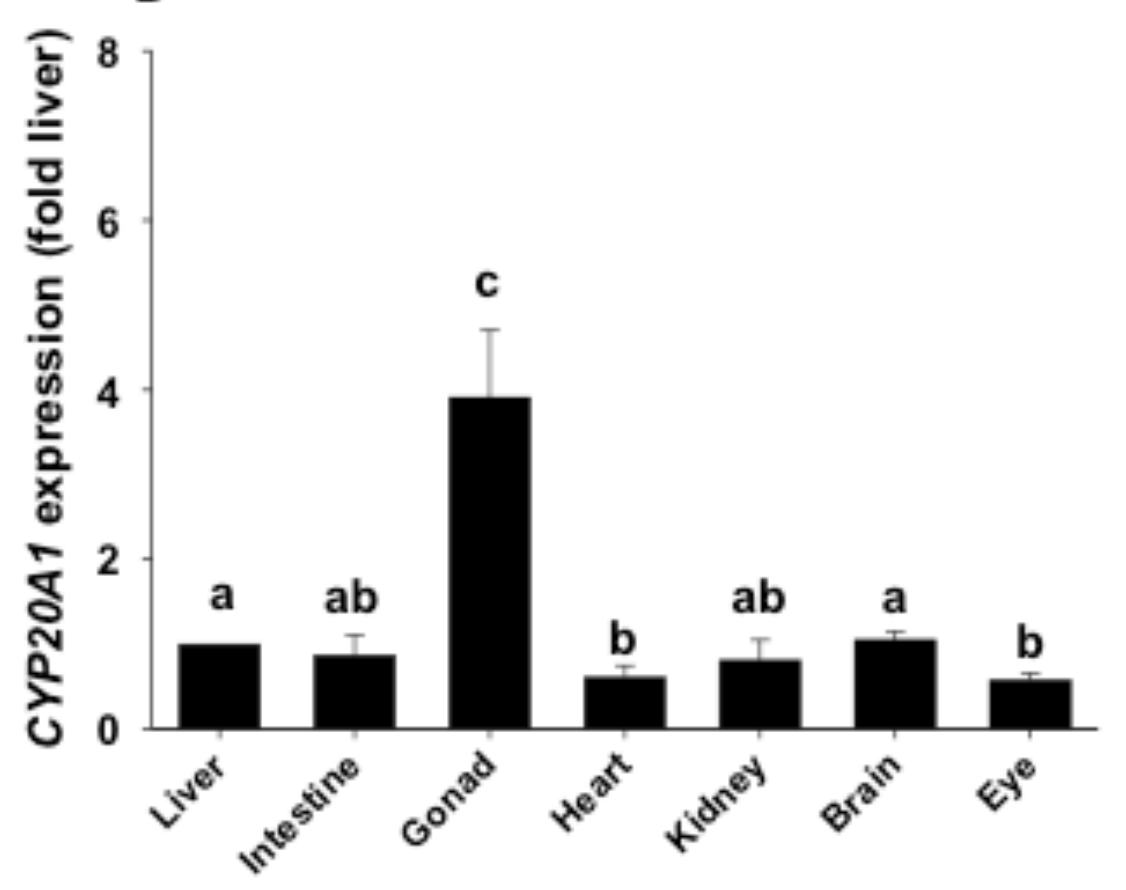

abc 
A

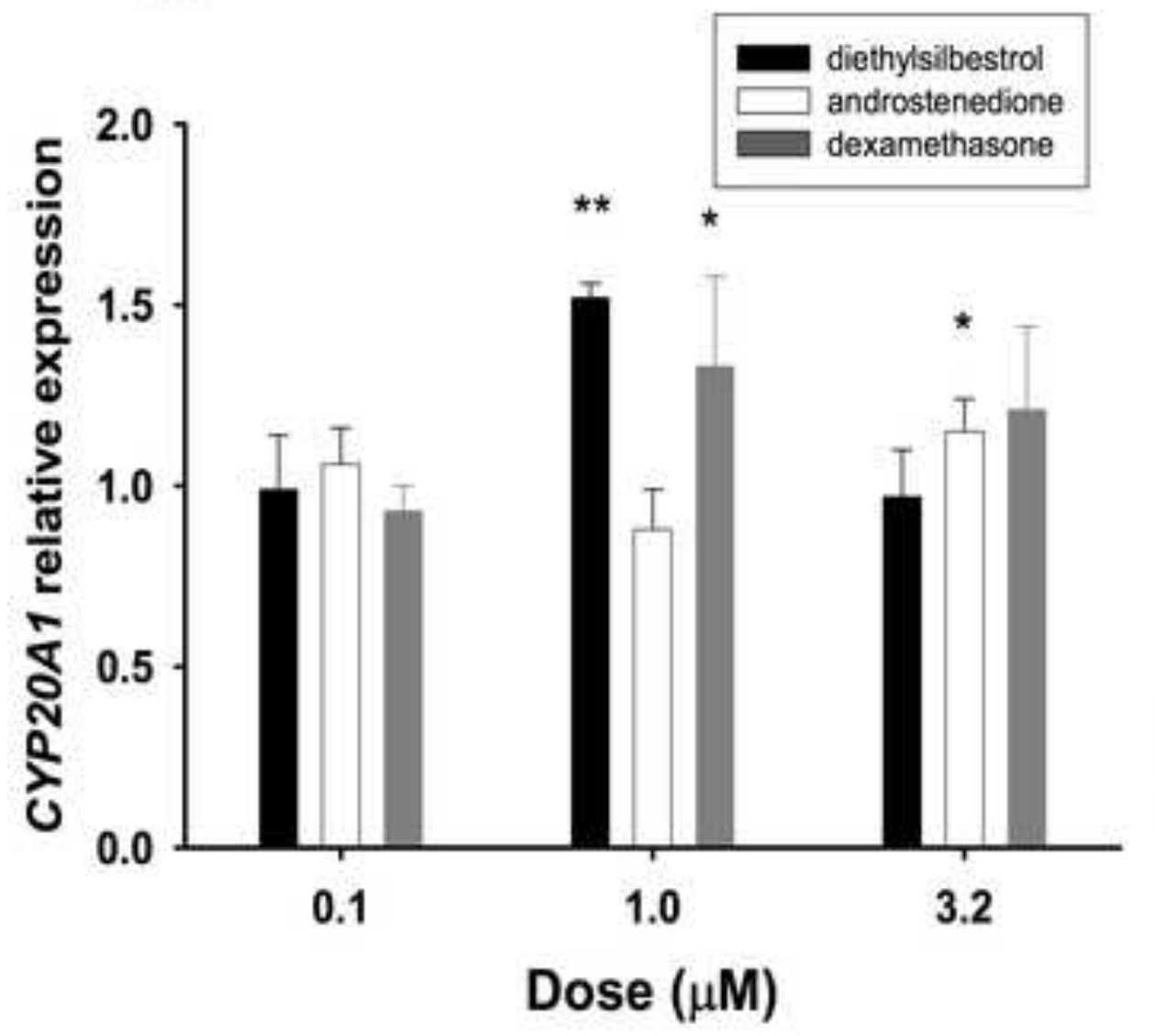

B

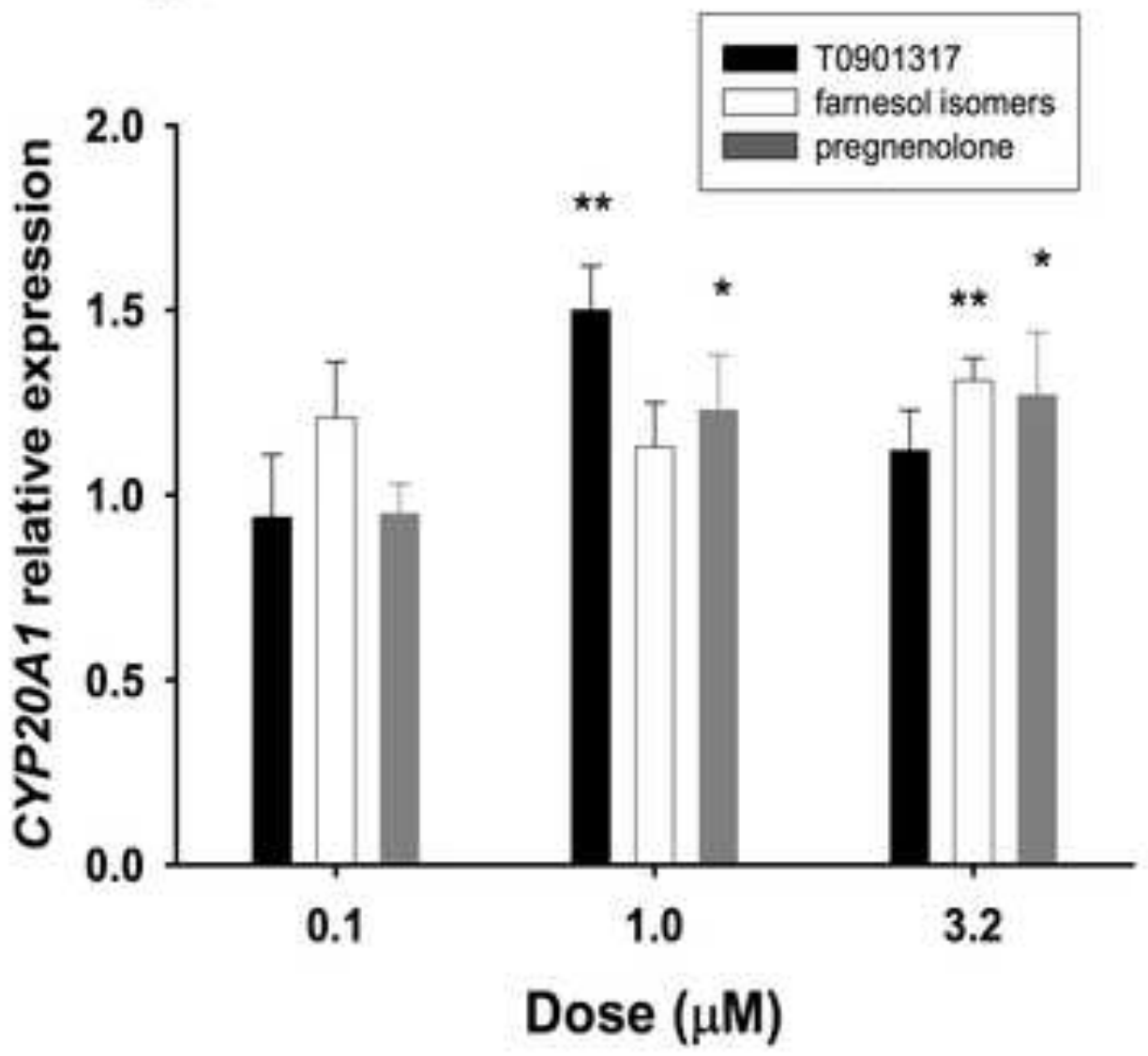




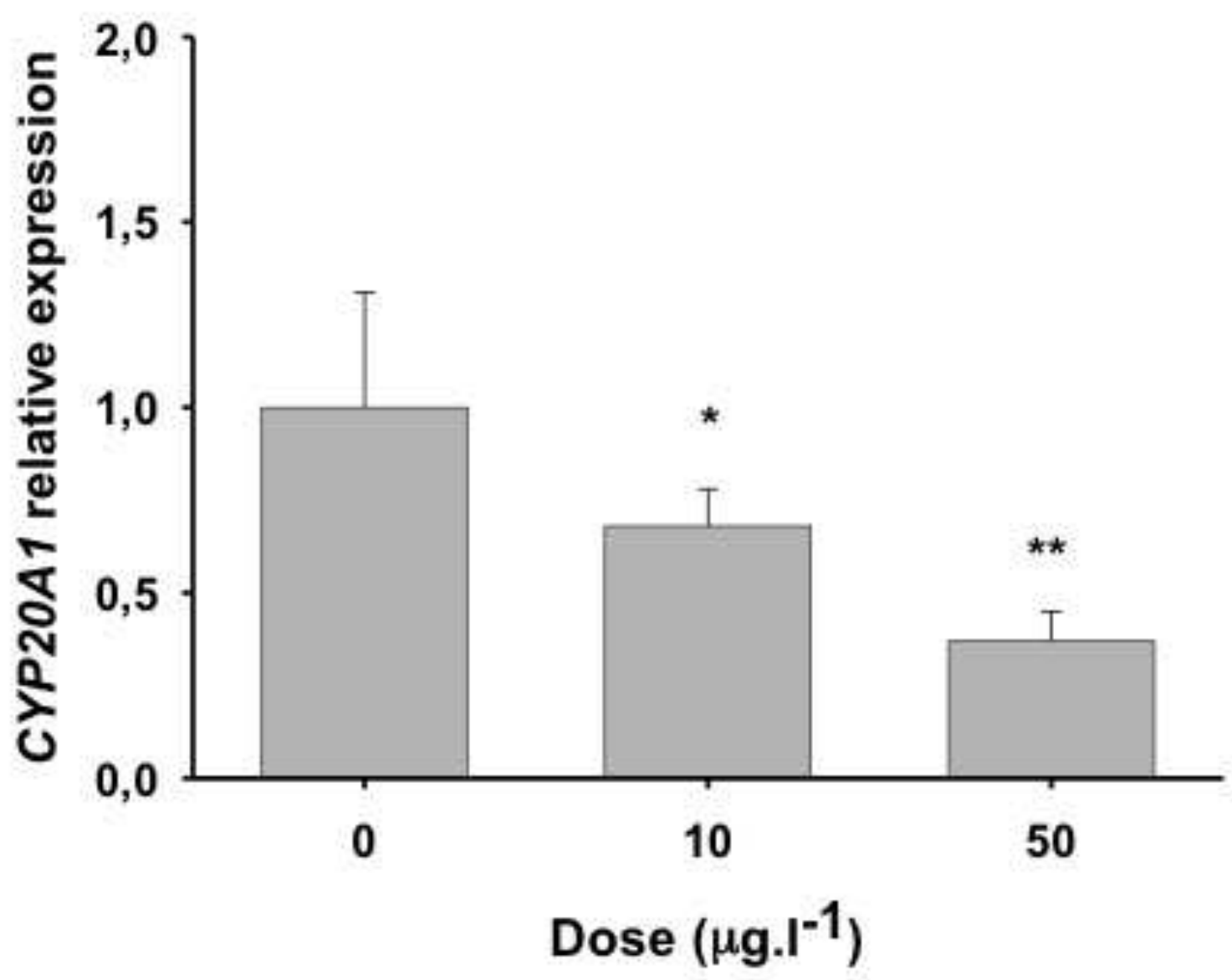




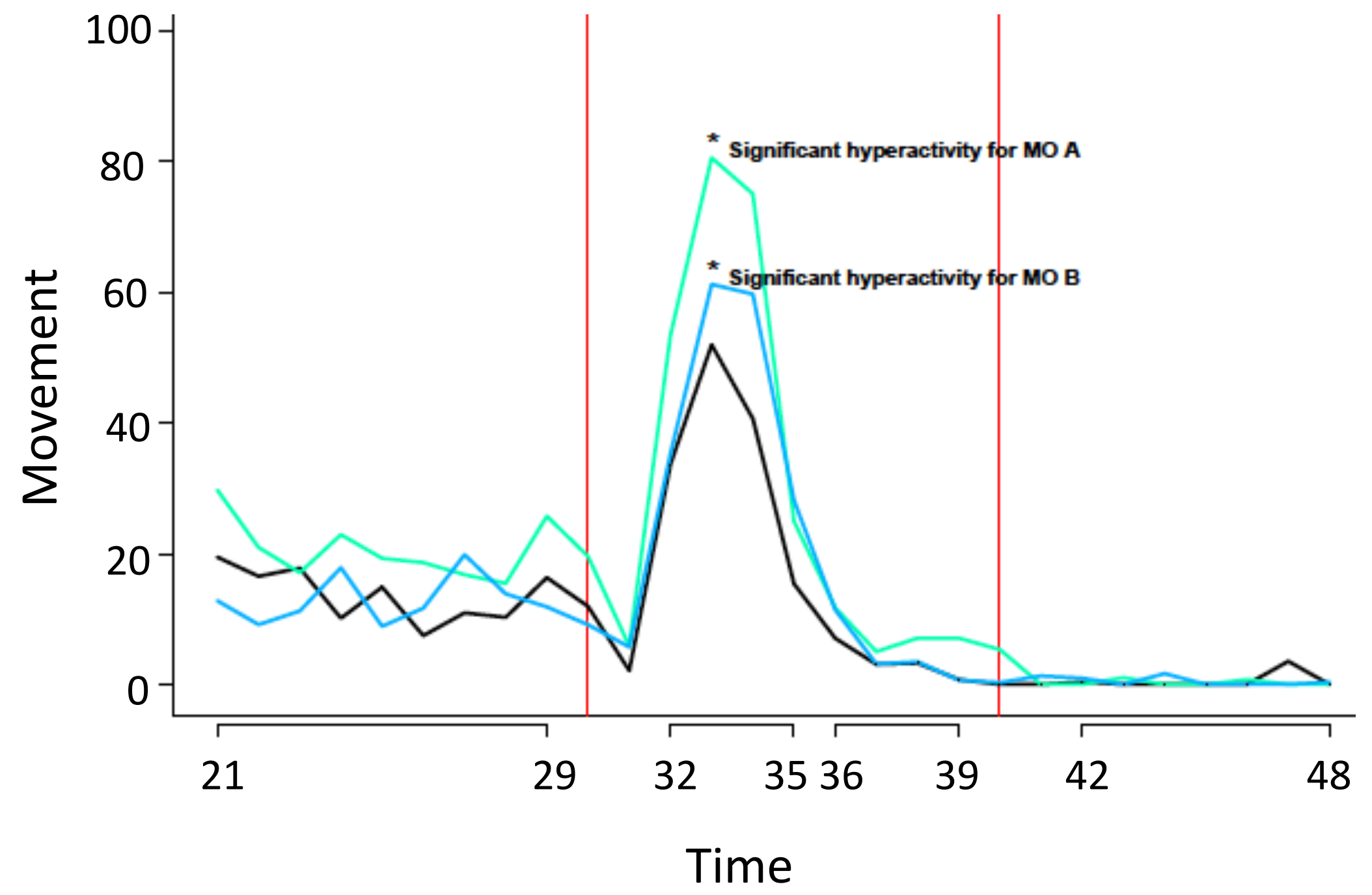


A
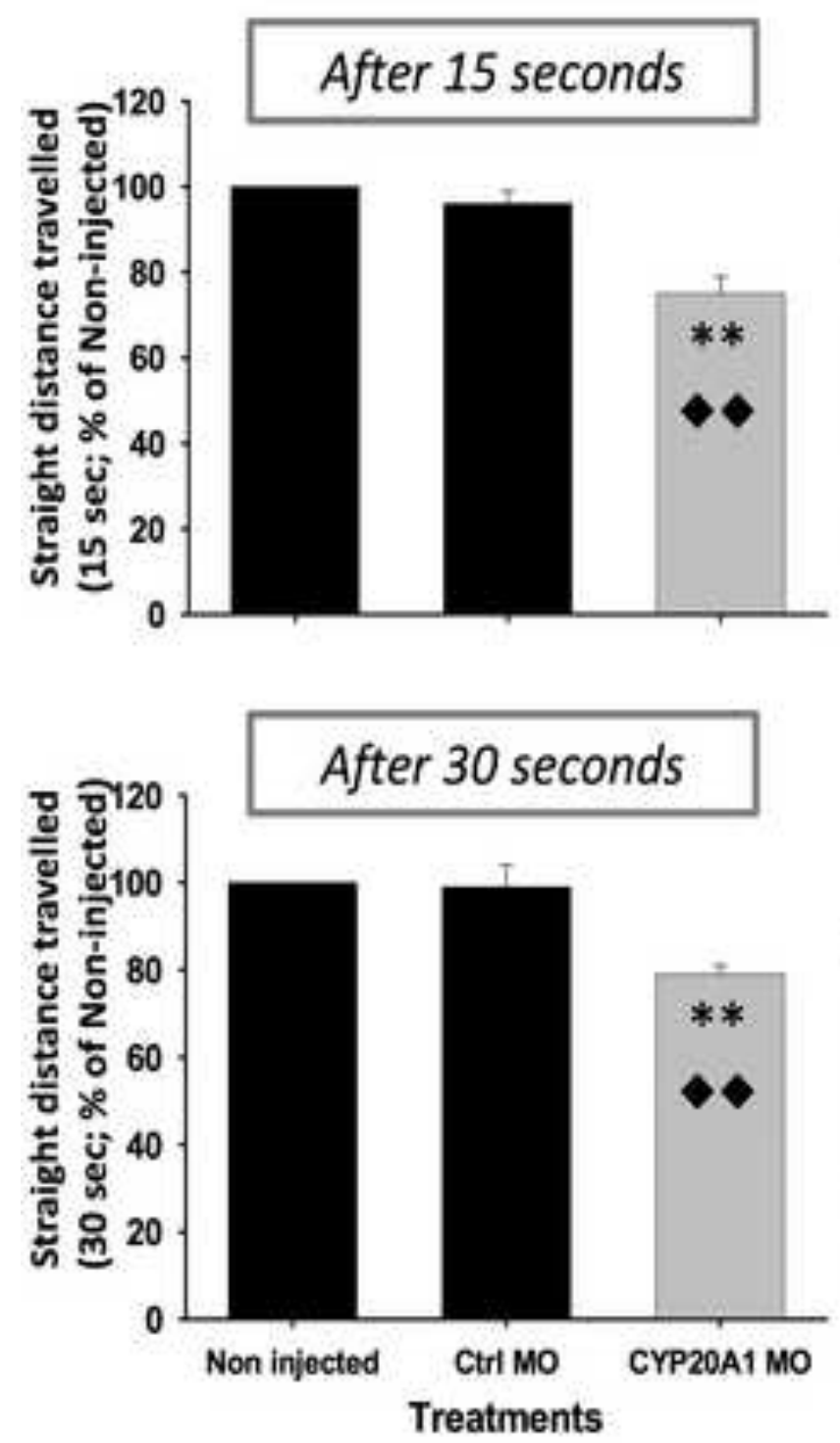

B
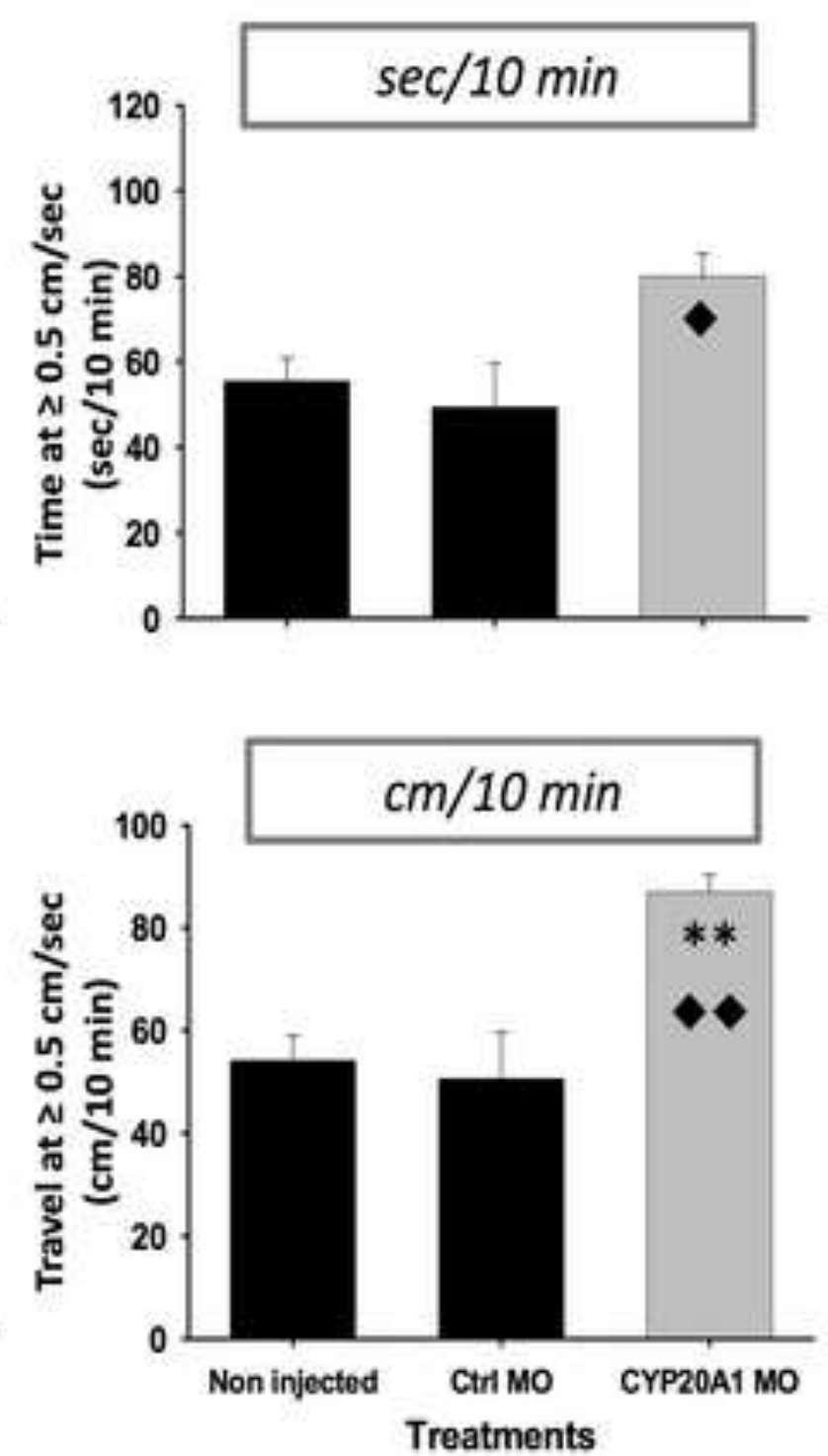

C
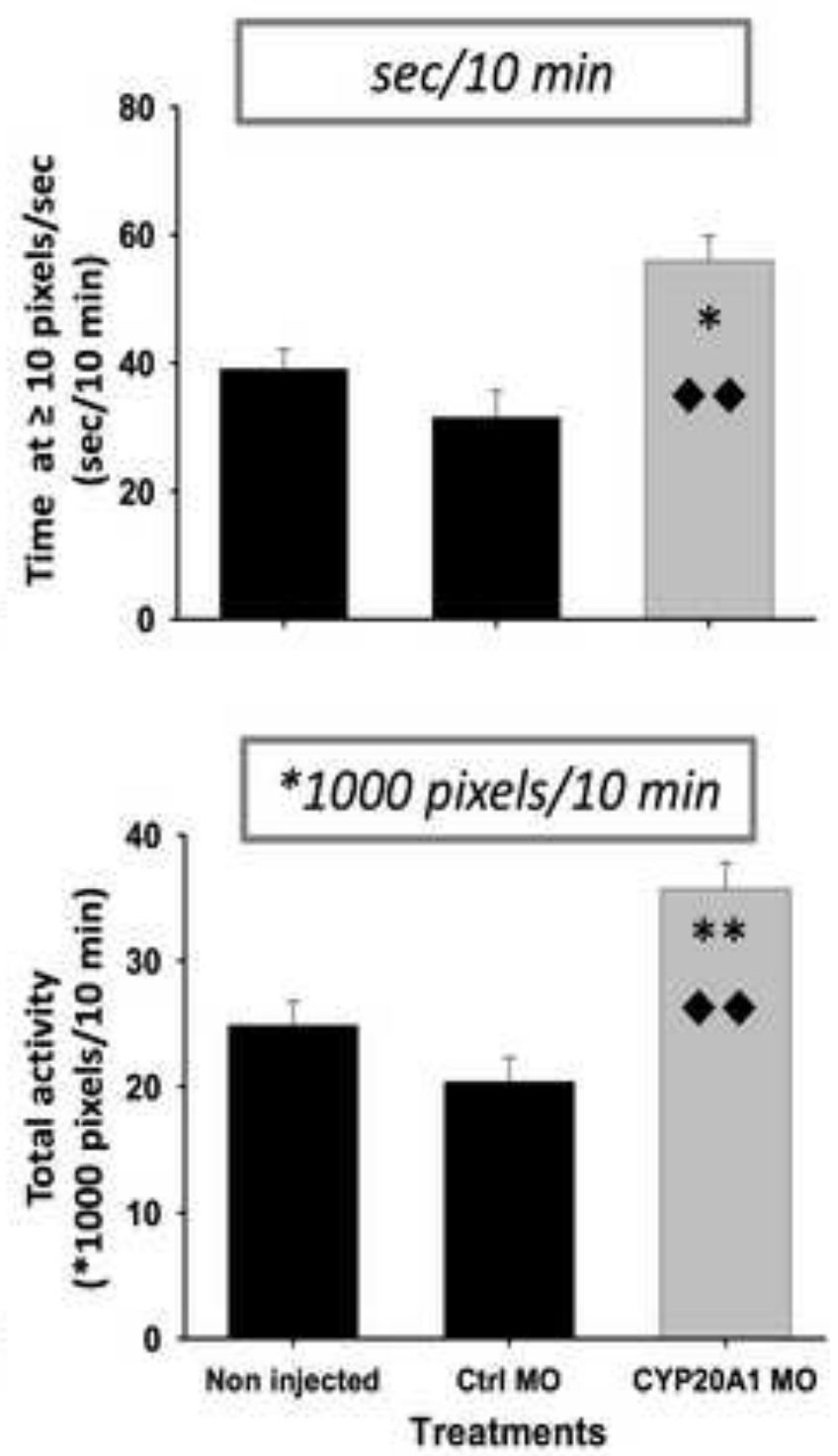